\title{
Dispersive estimates for Schrödinger operators in dimension two
}

\author{
W. Schlag*
}

\section{Introduction}

The purpose of this paper is to prove the following result.

Theorem 1. Let $V: \mathbb{R}^{2} \rightarrow \mathbb{R}$ be a measurable function such that $|V(x)| \leq C(1+$ $|x|)^{-\beta}, \beta>3$. Assume in addition that zero is a regular point of the spectrum of $H=-\triangle+V$. Then

$$
\left\|e^{i t H} P_{a c}(H) f\right\|_{\infty} \leq C|t|^{-1}\|f\|_{1}
$$

for all $f \in L^{1}\left(\mathbb{R}^{2}\right)$.

The definition of zero being a regular point amounts to the following, see Jensen, Nenciu [JenNen and Definition 7 below: Let $V \not \equiv 0$ and set $U=\operatorname{sign} V, v=|V|^{\frac{1}{2}}$. Let $P_{v}$ be the orthogonal projection onto $v$ and set $Q=I-P_{v}$. Finally, let

$$
\left(G_{0} f\right)(x):=-\frac{1}{2 \pi} \int_{\mathbb{R}^{2}} \log |x-y| f(y) d y .
$$

Then zero is regular iff $Q\left(U+v G_{0} v\right) Q$ is invertible on $Q L^{2}\left(\mathbb{R}^{2}\right)$.

Jensen and Nenciu study $\operatorname{ker}\left[Q\left(U+v G_{0} v\right) Q\right]$ on $Q L^{2}\left(\mathbb{R}^{2}\right)$. It can be completely described in terms of solutions $\Psi$ of $H \Psi=0$. In particular, its dimension is at most three plus the dimension of the zero energy eigenspace, see Theorem 6.2 and Lemma 6.4 in JenNen. The extra three dimensions here are called resonances. Hence, the requirement that zero is a regular point is the analogue of the usual condition that zero is neither an eigenvalue nor a resonance of $H$.

As far as the spectral properties of $H$ are concerned, we note that under the hypotheses of Theorem 1 the spectrum of $H$ on $[0, \infty)$ is purely absolutely continuous, and that the spectrum is pure point on $(-\infty, 0)$ with at most finitely many eigenvalues of finite multiplicities. The latter follows for example from Stoiciu [Sto], who obtained Birman-Schwinger type bounds in the case of two dimensions.

Theorem 1 appears to be the first $L^{1} \rightarrow L^{\infty}$ bound with $|t|^{-1}$ decay in $\mathbb{R}^{2}$. Yajima Yaj and Jensen, Yajima JenYaj proved the $L^{p}\left(\mathbb{R}^{2}\right)$ boundedness of the

\footnotetext{
${ }^{*}$ The author was partially supported by the NSF grant DMS-0300081 and a Sloan Fellowship
} 
wave operators under stronger decay assumptions on $V(x)$, but only for $1<p<$ $\infty$. Hence their result does not imply Theorem 11. Local $L^{2}$ decay was studied by Murata $\underline{M u r}$, but he does not consider $L^{1} \rightarrow L^{\infty}$ estimates.

The first $L^{1}\left(\mathbb{R}^{n}\right) \rightarrow L^{\infty}\left(\mathbb{R}^{n}\right)$ bounds for $e^{i t H}$ with $|t|^{-\frac{n}{2}}$ decay were obtained by Journé, Soffer, and Sogge JouSofSog. However, their argument depends on the fact that $t^{-\frac{n}{2}}$ is integrable at $t=\infty$, and thus only applies for $n \geq 3$. In dimension $n=1$ Weder [Wed] obtained the $|t|^{-\frac{1}{2}}$-decay under some conditions on $V$ which were then relaxed by Goldberg and the author GolSch. However, the case $n=2$ remained open.

As usual, the proof of Theorem 1breaks up into two regimes: energies bigger than $\lambda_{1}$ and energies in $\left(0, \lambda_{1}\right)$. Here $\lambda_{1}>0$ is some small constant. The corresponding statements are Propositions 4 and 11 below. Theorem 11 then follows by combining these two Propositions. For energies in $\left(0, \lambda_{1}\right)$ we use the recent results of Jensen and Nenciu JJenNen on expansions of the resolvent $\left(H-\left(\lambda^{2} \pm i 0\right)\right)^{-1}$ for $\lambda$ close to zero. Since we require somewhat finer estimates on various error terms, we give a complete derivation of this expansion. However, we emphasize that this derivation is of course merely a variant of a special case of the expansions in JenNen. In fact, the main achievement of Jensen and Nenciu is to determine the expansion of the perturbed resolvent in the presence of resonances and eigenvalues at zero.

\section{Energies separated from zero}

The main purpose of this section is to prove the dispersive estimate for the evolution restricted to energies $\left[\lambda_{1}, \infty\right), \lambda_{1}>0$. This will be accomplished by an expansion of the perturbed resolvent into a finite Born series, see (18) and (19). The main difficulty is to obtain the dispersive bound for each term of the Born series. This is done in Lemma 3 below. For the remainder (19) in the Born expansion, which still contains the perturbed resolvent, we use the limiting absorption principle. The approach in this section is modelled after that in GolSch], which in turn had its origins in the work of Rodnianski and the author RodSch.

Lemma 2 is a variant of the standard stationary phase method. In what follows, the notation $x \lesssim y$ means that $x \leq C y$ for some constant $C$.

Lemma 2. Let $\phi(0)=\phi^{\prime}(0)=0$ and $1 \leq \phi^{\prime \prime} \leq C$. Then

$$
\left|\int_{-\infty}^{\infty} e^{i t \phi(x)} a(x) d x\right| \lesssim \delta^{2} \int\left(\frac{|a(x)|}{\delta^{2}+|x|^{2}}+\chi_{[|x|>\delta]} \frac{\left|a^{\prime}(x)\right|}{|x|}\right) d x
$$

where $\delta=|t|^{-\frac{1}{2}}$. 
Proof. With $\eta$ being a standard cut-off one has

$$
\begin{aligned}
\left|\int_{-\infty}^{\infty} e^{i t \phi(x)} a(x) d x\right| & \leq\left|\int_{-\infty}^{\infty} e^{i t \phi(x)} a(x) \eta(x / \delta) d x\right| \\
& \lesssim\left|\int_{-\infty}^{\infty} e^{i t \phi(x)} a(x)(1-\eta(x / \delta)) d x\right| \\
& \lesssim \int_{|x|<\delta}|a(x)| d x+\delta^{2} \int\left|\left(\frac{a(x)(1-\eta(x / \delta))}{\phi^{\prime}(x)}\right)^{\prime}\right| d x
\end{aligned}
$$

as claimed.

It is well-known that

$$
R_{0}^{ \pm}\left(\lambda^{2}\right)(x, y)=\left(-\Delta-\left(\lambda^{2} \pm i 0\right)\right)^{-1}(x, y)= \pm \frac{i}{4} H_{0}^{ \pm}(\lambda|x-y|)
$$

where $H_{0}^{ \pm}$are the Hankel functions of order zero with $H_{0}^{-}=\overline{H_{0}^{+}}$. They have the form

$$
H_{0}^{+}(y)=e^{i(y-1)} \omega(y) \chi_{[y>1]}+\omega(y) \chi_{[0<y<1]}
$$

and satisfy the bounds $|\omega(y)| \lesssim|y|^{-\frac{1}{2}}$ if $y \gtrsim 1$ and $|\omega(y)| \lesssim|\log y|$ of $0<y<\frac{1}{2}$. Moreover, one has for all positive integers $\nu$,

$$
\begin{aligned}
\left|\omega^{(\nu)}(y)\right| & \lesssim|y|^{-\nu-\frac{1}{2}} \quad \text { if } \quad y \gtrsim 1 \\
\left|\omega^{(\nu)}(y)\right| & \lesssim|y|^{-\nu} \quad \text { if } \quad 0<y<1 .
\end{aligned}
$$

Set $\omega_{+}(y)=\chi_{1}\left(y / y_{0}\right) \omega(y)$ where $\chi_{1}(y)=0$ if $y \leq 1$ and $=1$ if $y \geq 2$. Here $y_{0} \gg 1$ is a fixed constant. Define $\omega_{-}(y)$ via $\omega=\omega_{+}+\omega_{-}$, i.e., $\omega_{-}(y)=\left(1-\chi_{1}\left(y / y_{0}\right)\right) \omega(y)$ (in Section 3 the functions $\omega_{+}$and $\omega_{-}$will take on a different meaning, not to be confused with the one here). Let

$$
\|V\|_{\mathcal{K}}:=\sup _{x \in \mathbb{R}^{2}} \int_{\mathbb{R}^{2}}\left(1+\log ^{-}|x-y|\right)^{2}|V(y)| d y
$$

where $\log ^{-} u=-\chi_{[0<u<1]} \log u$. Finally, pick a cut-off $\chi_{2}$ so that $\chi_{2}(y)=1$ if $y \leq 1$ and $\chi_{2}(y)=0$ if $y \geq 2$. The following lemma is one of the two main technical ingredients of the high energy part. We urge the reader not to be distracted by the technical appearance of the proof. Indeed, the bound (3) can be derived heuristically as an immediate consequence of stationary phase. However, some cases do need to be distinguished due to various cut-offs in the integrand. 
Lemma 3. Assume $\|V\|_{\mathcal{K}}<\infty$. Let $\{1,2, \ldots, m\}=J \cup J^{*}$ be a partition. Then

$$
\begin{aligned}
& \sup _{\substack{L \geq 1 \\
x_{0}, x_{m} \in \mathbb{R}^{2}}} \int_{\mathbb{R}^{2(m-1)}} \mid \int_{0}^{\infty} \lambda e^{i\left(t \lambda^{2} \pm \lambda \sum_{j \in J}\left|x_{j-1}-x_{j}\right|\right)} \chi_{1}(\lambda) \chi_{2}(\lambda / L) \prod_{j \in J} \omega_{+}\left(\lambda\left|x_{j-1}-x_{j}\right|\right) \\
& \left.\prod_{\ell \in J^{*}} \omega_{-}\left(\lambda\left|x_{\ell-1}-x_{\ell}\right|\right) d \lambda\left|\prod_{k=1}^{m-1}\right| V\left(x_{k}\right)\left|d x_{1} \ldots d x_{m-1} \lesssim\right| t\right|^{-1}\|V\|_{\mathcal{K}}^{m-1}
\end{aligned}
$$

with a constant that only depends on $m$.

Proof. The heuristic reason for this bound is as follows: Let $d_{j}=\left|x_{j}-x_{j-1}\right|$ and $s=\sum_{j \in J} d_{j}$. If there is a critical point of the phase, then it is $\lambda_{0}=\frac{s}{2 t}$ (assuming $t>0)$. We may assume that $\lambda_{0} \gtrsim 1$, otherwise the integrand vanishes at $\lambda_{0}$. Using stationary phase, the inner integral is then bounded by

$$
t^{-\frac{1}{2}} \lambda_{0}\left(\lambda_{0} s\right)^{-\frac{1}{2}} \prod_{\ell \in J^{*}} \log ^{-}\left(\lambda_{0} d_{\ell}\right) \lesssim t^{-1} \prod_{\ell \in J^{*}} \log ^{-}\left(d_{\ell}\right) .
$$

Inserting this bound into (3) then yields the desired result by an application of Cauchy-Schwartz, see (5) below.

To make this rigorous, we start off integrating by parts: Then

$$
\begin{aligned}
& |t|\left|\int_{0}^{\infty} \lambda e^{i\left(t \lambda^{2} \pm \lambda s\right)} \chi_{1}(\lambda) \chi_{2}(\lambda / L) \prod_{j \in J} \omega_{+}\left(\lambda d_{j}\right) \prod_{\ell \in J^{*}} \omega_{-}\left(\lambda d_{\ell}\right) d \lambda\right| \\
& \lesssim\left|\int_{0}^{\infty} e^{i\left(t \lambda^{2} \pm \lambda s\right)} \chi_{1}^{\prime}(\lambda) \chi_{2}(\lambda / L) \prod_{j \in J} \omega_{+}\left(\lambda d_{j}\right) \prod_{\ell \in J^{*}} \omega_{-}\left(\lambda d_{\ell}\right) d \lambda\right| \\
& +\frac{1}{L}\left|\int_{0}^{\infty} e^{i\left(t \lambda^{2} \pm \lambda s\right)} \chi_{1}(\lambda) \chi_{2}^{\prime}(\lambda / L) \prod_{j \in J} \omega_{+}\left(\lambda d_{j}\right) \prod_{\ell \in J^{*}} \omega_{-}\left(\lambda d_{\ell}\right) d \lambda\right| \\
& +s\left|\int_{0}^{\infty} e^{i\left(t \lambda^{2} \pm \lambda s\right)} \chi_{1}(\lambda) \chi_{2}(\lambda / L) \prod_{j \in J} \omega_{+}\left(\lambda d_{j}\right) \prod_{\ell \in J^{*}} \omega_{-}\left(\lambda d_{\ell}\right) d \lambda\right| \\
& +\sum_{k \in J} d_{k}\left|\int_{0}^{\infty} e^{i\left(t \lambda^{2} \pm \lambda s\right)} \chi_{1}(\lambda) \chi_{2}(\lambda / L) \omega_{+}^{\prime}\left(\lambda d_{k}\right) \prod_{\substack{j \in J \\
j \neq k}} \omega_{+}\left(\lambda d_{j}\right) \prod_{\ell \in J^{*}} \omega_{-}\left(\lambda d_{\ell}\right) d \lambda\right| \\
& +\sum_{k \in J^{*}} d_{k}\left|\int_{0}^{\infty} e^{i\left(t \lambda^{2} \pm \lambda s\right)} \chi_{1}(\lambda) \chi_{2}(\lambda / L) \prod_{j \in J} \omega_{+}\left(\lambda d_{j}\right) \omega_{-}^{\prime}\left(\lambda d_{k}\right) \prod_{\ell \in J^{*}} \omega_{-}\left(\lambda d_{\ell}\right) d \lambda\right| \\
& =: A^{ \pm}+B^{ \pm}+C^{ \pm}+D^{ \pm}+E^{ \pm} .
\end{aligned}
$$


Let $k(x, y):=1+\log ^{-}|x-y|$. Then since $\log ^{-}$is decreasing,

$$
\begin{aligned}
\left|A^{ \pm}\right| & \lesssim \int\left|\chi_{1}^{\prime}(\lambda)\right| \prod_{j \in J^{*}}\left|\omega_{-}\left(\lambda d_{j}\right)\right| d \lambda \\
& \lesssim \int\left|\chi_{1}^{\prime}(\lambda)\right| \prod_{j \in J^{*}}\left(1+\log ^{-}\left(\lambda d_{j}\right)\right) d \lambda \lesssim \int\left|\chi_{1}^{\prime}(\lambda)\right| \prod_{j \in J^{*}}\left(1+\log ^{-}\left(d_{j}\right)\right) d \lambda \\
& \lesssim \prod_{j \in J^{*}} k\left(x_{j-1}, x_{j}\right) .
\end{aligned}
$$

Hence the contribution of $A^{ \pm}$to (3) is

$$
\begin{aligned}
& \lesssim \int_{\mathbb{R}^{2(m-1)}} \prod_{j=1}^{m} k\left(x_{j-1}, x_{j}\right) \prod_{j=1}^{m-1}\left|V\left(x_{j}\right)\right| d x_{1} \ldots d x_{m-1} \\
& \lesssim \int\left(k^{2}\left(x_{0}, x_{1}\right)\left|V\left(x_{1}\right)\right|+\left|V\left(x_{1}\right)\right| k^{2}\left(x_{1}, x_{2}\right)\right) \prod_{j=2}^{m-1}\left|V\left(x_{j}\right)\right| k\left(x_{j}, x_{j+1}\right) d x_{1} \ldots d x_{m-1} \\
& \lesssim\|V\|_{\mathcal{K}}^{m-1} .
\end{aligned}
$$

For the remainder of the proof we set

$$
P_{*}=\prod_{j \in J^{*}} k\left(x_{j-1}, x_{j}\right)
$$

with the understanding that $P_{*}=1$ if $J^{*}=\emptyset$. Similarly, for $L \geq 1$, one has that

$$
\left|B^{ \pm}\right| \lesssim \frac{1}{L} \int_{0}^{\infty}\left|\chi_{2}^{\prime}(\lambda / L)\right| \prod_{j \in J^{*}}\left|\omega_{-}\left(\lambda d_{j}\right)\right| d \lambda \lesssim P_{*} .
$$

Hence the contribution by $B^{ \pm}$to (3) is again $\lesssim\|V\|_{\mathcal{K}}^{m-1}$. The terms $D^{ \pm}, E^{ \pm}$are also easy to deal with. Indeed, one has

$$
\begin{aligned}
\left|D^{ \pm}\right| & \lesssim \sum_{k \in J} d_{k} \int_{0}^{\infty}\left(1+\lambda d_{k}\right)^{-\frac{3}{2}} \prod_{\substack{j \in J \\
j \neq k}}\left(1+\lambda d_{j}\right)^{-\frac{1}{2}} d \lambda P_{*} \\
& =\int_{0}^{\infty}\left[-2 \prod_{j \in J}\left(1+\lambda d_{j}\right)^{-\frac{1}{2}}\right]^{\prime} d \lambda P_{*}=2 P_{*} .
\end{aligned}
$$

As far as $E^{ \pm}$is concerned, we conclude similarly that (with some small constant $c>0)$

$$
\begin{aligned}
\left|E^{ \pm}\right| & \lesssim \sum_{k \in J^{*}} d_{k} \int_{1}^{\infty}\left(d_{k} \lambda\right)^{-1} \chi_{\left[c \lambda d_{k} \leq 1\right]} \prod_{\substack{j \in J^{*} \\
j \neq k}} \log ^{-}\left(c \lambda d_{j}\right) d \lambda \\
& =\int_{1}^{\infty}\left[-\prod_{j \in J^{*}} \log ^{-}\left(c \lambda d_{j}\right)\right]^{\prime} d \lambda \lesssim P_{*} .
\end{aligned}
$$


We now apply Lemma 2 to $C^{-}$with $\phi_{-}(\lambda)=\lambda^{2}-\lambda \frac{s}{t}$ and

$$
a(\lambda)=a_{+}(\lambda) \prod_{j \in J^{*}} \omega_{-}\left(\lambda d_{j}\right), \quad a_{+}(\lambda)=\chi_{1}(\lambda) \chi_{2}(\lambda / L) \prod_{j \in J} \omega_{+}\left(\lambda d_{j}\right) .
$$

Note that

$$
\begin{aligned}
|a(\lambda)| & \lesssim a_{+}(\lambda) \prod_{j \in J^{*}} k\left(x_{j-1}, x_{j}\right)=a_{+}(\lambda) P_{*} \\
\left|a^{\prime}(\lambda)\right| & \lesssim\left|a_{+}^{\prime}(\lambda)\right| P_{*}+\sum_{j \in J^{*}}\left|a_{+}(\lambda)\right| \lambda^{-1} \chi_{\left[\lambda d_{j} \lesssim 1\right]} \prod_{\substack{k \in J^{*} \\
k \neq j}}\left(1+\log ^{-}\left(\lambda d_{k}\right)\right) .
\end{aligned}
$$

Set $g(y)=1+\log ^{-}(y)$ so that $g^{\prime}(y)=-\chi_{[0<y<1]} y^{-1}$. Define

$$
b(\lambda)=\prod_{j \in J^{*}} g\left(c \lambda d_{j}\right) \text { with some small } c>0 .
$$

Then $0<b(\lambda) \lesssim \prod_{j \in J^{*}} k\left(x_{j-1}, x_{j}\right)$ for $\lambda \gtrsim 1$ and

$$
\left|a^{\prime}(\lambda)\right| \lesssim\left|a_{+}^{\prime}(\lambda)\right| P_{*}+\left|a_{+}(\lambda)\right|\left|b^{\prime}(\lambda)\right| \lesssim\left|a_{+}^{\prime}(\lambda)\right| P_{*}+a_{0}(\lambda)\left(-b^{\prime}(\lambda)\right)
$$

where $a_{0}(\lambda)=\chi_{1}(\lambda) \chi_{2}(\lambda / L) \prod_{j \in J}\left(1+\lambda d_{j}\right)^{-\frac{1}{2}}$.

One has $\phi_{-}^{\prime}\left(\lambda_{0}\right)=0$ for $\lambda_{0}=\frac{s}{2 t}$. We first assume that $\chi_{1}\left(\lambda_{0}\right) \neq 0$ as well as $\lambda_{0} \in \operatorname{supp}\left(\omega_{+}\left(d_{j} \cdot\right)\right)$ for each $j \in J$. These assumptions translate into $\lambda_{0} \gtrsim 1$ and $\min _{j \in J} \lambda_{0} d_{j} \gg 1$. The latter condition implies that $\lambda_{0}^{2}=\frac{s \lambda_{0}}{2 t} \gg t^{-1}$ and thus $\lambda_{0} \gg \delta=|t|^{-\frac{1}{2}}$. Next, we use Lemma 2 to bound $\left|C^{-}\right|$. On the one hand,

$$
\begin{aligned}
& s \delta^{2} \int \frac{|a(\lambda)| P_{*}^{-1}}{\delta^{2}+\left|\lambda-\lambda_{0}\right|^{2}} d \lambda \\
\lesssim & s \delta^{2} \int_{\lambda_{0}-\delta}^{\infty} \frac{(1+\lambda s)^{-\frac{1}{2}}}{\delta^{2}+\left(\lambda-\lambda_{0}\right)^{2}} d \lambda+s \delta^{2} \int_{1}^{\lambda_{0}-\delta} \frac{(1+\lambda s)^{-\frac{1}{2}}}{\left(\lambda-\lambda_{0}\right)^{2}} d \lambda \\
\lesssim & s \delta^{2}\left(1+\lambda_{0} s\right)^{-\frac{1}{2}} \int_{\lambda_{0}-\delta}^{\infty} \frac{1}{\delta^{2}+\left(\lambda-\lambda_{0}\right)^{2}} d \lambda+\sqrt{s} \delta^{2} \int_{1}^{\lambda_{0}-\delta} \frac{d \lambda}{\sqrt{\lambda}\left(\lambda_{0}-\lambda\right)^{2}} \\
\lesssim & \sqrt{s} \lambda_{0}^{-\frac{1}{2}} \delta+\sqrt{s} \delta^{2} \lambda_{0}^{-\frac{3}{2}} \\
\lesssim & \sqrt{\frac{s}{t}} \lambda_{0}^{-\frac{1}{2}}+\sqrt{\frac{s}{t}} \lambda_{0}^{-\frac{1}{2}} \delta \lambda_{0}^{-1} \lesssim 1
\end{aligned}
$$


On the other hand, see (10), an integration by parts yields

$$
\begin{aligned}
& s \delta^{2} \int_{\left|\lambda-\lambda_{0}\right|>\delta} \frac{\left|a^{\prime}(\lambda)\right|}{\left|\lambda-\lambda_{0}\right|} d \lambda \\
& \lesssim s \delta^{2} \int_{\left|\lambda-\lambda_{0}\right|>\delta} \frac{\left|a_{+}^{\prime}(\lambda)\right| P_{*}}{\left|\lambda-\lambda_{0}\right|} d \lambda+s \delta^{2} \int_{\left|\lambda-\lambda_{0}\right|>\delta} \frac{a_{0}(\lambda)\left(-b^{\prime}(\lambda)\right)}{\left|\lambda-\lambda_{0}\right|} d \lambda \\
& \lesssim s \delta^{2} \int_{\left|\lambda-\lambda_{0}\right|>\delta} \frac{\left|a_{+}^{\prime}(\lambda)\right| P_{*}}{\left|\lambda-\lambda_{0}\right|} d \lambda+s \delta^{2} \int_{\left|\lambda-\lambda_{0}\right|>\delta} \frac{a_{0}^{\prime}(\lambda) b(\lambda)}{\left|\lambda-\lambda_{0}\right|} d \lambda \\
& +s \delta^{2} \int_{1}^{\lambda_{0}-\delta} \frac{a_{0}(\lambda) b(\lambda)}{\left(\lambda-\lambda_{0}\right)^{2}} d \lambda+\left.s \delta^{2} \frac{a_{0}(\lambda) b(\lambda)}{\left|\lambda-\lambda_{0}\right|}\right|_{\lambda-\lambda_{0}= \pm \delta} .
\end{aligned}
$$

By the estimates leading up to (11) one has (13) $\lesssim P_{*}$. On the other hand,

$$
\begin{gathered}
\text { (12) } \lesssim s \delta^{2} P_{*} \int_{\lambda_{0}+\delta}^{\infty}\left(\frac{1}{L}\left|\chi_{2}^{\prime}(\lambda / L)\right|(1+\lambda s)^{-\frac{1}{2}}+\lambda^{-1}(1+\lambda s)^{-\frac{1}{2}}\right) \frac{d \lambda}{\lambda-\lambda_{0}} \\
+s \delta^{2} P_{*} \int_{1}^{\lambda_{0}-\delta}\left[\left|\chi_{1}^{\prime}(\lambda)\right|(1+s)^{-\frac{1}{2}}+\frac{\left|\chi_{2}^{\prime}(\lambda / L)\right|}{L(1+\lambda s)^{\frac{1}{2}}}\right. \\
\left.+\sum_{j \in J} d_{j}\left(1+\lambda d_{j}\right)^{-\frac{3}{2}} \prod_{\substack{k \in J \\
k \neq j}}\left(1+\lambda d_{k}\right)^{-\frac{1}{2}}\right] \frac{d \lambda}{\lambda_{0}-\lambda} .
\end{gathered}
$$

It will be convenient to resum the expression on the right-hand side of (14) by rewriting it as a derivative. This yields

$$
\text { (14) } \begin{aligned}
& \lesssim \sqrt{s} \delta \lambda_{0}^{-\frac{1}{2}} P_{*}+s \delta^{2} P_{*} \int_{1}^{\lambda_{0}-\delta}\left[-\prod_{k \in J}\left(1+\lambda d_{k}\right)^{-\frac{1}{2}}\right]^{\prime} \frac{d \lambda}{\lambda_{0}-\lambda} \\
& \lesssim P_{*}+s \delta^{2} P_{*} \int_{1}^{\lambda_{0}-\delta} \prod_{k \in J}\left(1+\lambda d_{k}\right)^{-\frac{1}{2}} \frac{d \lambda}{\left(\lambda_{0}-\lambda\right)^{2}} \\
& +\left.s \delta^{2} P_{*} \prod_{k \in J}\left(1+\lambda d_{k}\right)^{-\frac{1}{2}} \frac{1}{\lambda_{0}-\lambda}\right|_{\lambda=1} \\
& \lesssim P_{*}+\sqrt{s} \delta^{2} P_{*} \int_{1}^{\lambda_{0}-\delta} \frac{d \lambda}{\lambda^{\frac{1}{2}}\left(\lambda_{0}-\lambda\right)^{2}}+s \delta^{2} \lambda_{0}^{-1} P_{*} \\
& \lesssim P_{*}+\left(\sqrt{s} \delta^{2} \lambda_{0}^{-\frac{3}{2}}+\sqrt{s} \delta \lambda_{0}^{-\frac{1}{2}}\right) P_{*} \lesssim P_{*} .
\end{aligned}
$$

In view of the preceding, $\left|C^{-}\right| \lesssim P_{*}$ provided $\lambda_{0} \gtrsim 1$ and $\min _{j \in J} \lambda_{0} d_{j} \gg 1$. This gives the desired contribution to (3).

Now suppose that $\lambda_{0} \gtrsim 1$ but $\min _{j \in J} \lambda_{0} d_{j} \lesssim 1$. Let $\mu=\min _{j \in J} d_{j}$ so that $\mu \lesssim \lambda_{0}^{-1}$. By construction, $\operatorname{supp}(a) \subset\left[C \mu^{-1}, \infty\right)$ for some large $C$. Therefore, $\lambda \gg \lambda_{0}$ as well 
as $\lambda-\lambda_{0} \gtrsim \lambda$ on $\operatorname{supp}(a)$. By Lemma 2 ,

$$
\begin{aligned}
\left|C^{-}\right| & \lesssim s \delta \max _{\left[\lambda_{0}-\delta, \lambda_{0}+\delta\right]}|a(\lambda)|+s \delta^{2} \int_{\lambda_{0}+\delta}^{\infty}\left[\frac{|a(\lambda)|}{\left(\lambda-\lambda_{0}\right)^{2}}+\frac{\left|a^{\prime}(\lambda)\right|}{\lambda-\lambda_{0}}\right] d \lambda \\
& \lesssim \sqrt{s} \delta \lambda_{0}^{-\frac{1}{2}} \chi_{\left[\delta \ll \lambda_{0}\right]} P_{*}+s \delta\left(1+s \mu^{-1}\right)^{-\frac{1}{2}} \chi_{\left[\delta \gtrsim \lambda_{0}\right]} P_{*}+s \delta^{2} \int_{\mu^{-1}}^{\infty}\left[\frac{|a(\lambda)|}{\lambda^{2}}+\frac{\left|a^{\prime}(\lambda)\right|}{\lambda}\right] d \lambda \\
& \lesssim P_{*}+s \delta^{2} \int_{\mu^{-1}}^{\infty}\left[\frac{|a(\lambda)|}{\lambda^{2}}+\frac{\left|a^{\prime}(\lambda)\right|}{\lambda}\right] d \lambda .
\end{aligned}
$$

To bound the integral we use

$$
\begin{aligned}
&|a(\lambda)| \lesssim(1+s \lambda)^{-\frac{1}{2}} \chi_{\left[\lambda>\mu^{-1}\right]} P_{*} \\
&\left|a^{\prime}(\lambda)\right| \lesssim \lambda^{-1}(1+s \lambda)^{-\frac{1}{2}} \chi_{\left[\lambda>\mu^{-1}\right]} P_{*},
\end{aligned}
$$

see (17) and (8). Therefore,

$$
\begin{aligned}
s \delta^{2} \int_{\mu^{-1}}\left[\frac{|a(\lambda)|}{\lambda^{2}}+\frac{\left|a^{\prime}(\lambda)\right|}{\lambda}\right] d \lambda & \lesssim s \delta^{2} P_{*} \int_{\mu^{-1}}^{\infty} \frac{(1+\lambda s)^{-\frac{1}{2}}}{\lambda^{2}} d \lambda \\
& \lesssim \sqrt{s} \delta^{2} \mu^{\frac{3}{2}} P_{*} \lesssim s \delta^{2} \mu P_{*}=\lambda_{0} \mu P_{*} \lesssim P_{*},
\end{aligned}
$$

where we used $\mu \leq s$ to pass to the second inequality in the second line. It remains to consider the case when $\lambda_{0} \ll 1$. Note that $a(\lambda)=0$ if $\min _{j \in J} \lambda d_{j} \lesssim 1$, which is the same as $\lambda \lesssim \mu^{-1}$. Also, $a(\lambda)=0$ is $\lambda \leq 1$. Then, again via Lemma 2, one obtains as in (15),

$$
\begin{aligned}
\left|C^{-}\right| & \lesssim \sqrt{s} \delta^{2} P_{*} \int_{1+\mu^{-1}}^{\infty} \lambda^{-\frac{5}{2}} d \lambda \lesssim \sqrt{s} \delta^{2}\left(1+\mu^{-1}\right)^{-\frac{3}{2}} P_{*} \\
& \lesssim \sqrt{s} \delta^{2} \chi_{[\mu>1]} P_{*}+\sqrt{s} \delta^{2} \mu^{\frac{3}{2}} \chi_{[\mu<1]} P_{*} \\
& \lesssim \frac{s}{t} P_{*}+\frac{s}{t} \mu \chi_{[\mu<1]} P_{*} \lesssim\left(\lambda_{0}+\lambda_{0} \mu \chi_{[\mu<1]}\right) P_{*} \lesssim P_{*} .
\end{aligned}
$$

The lemma is proved.

Proposition 4. Assume that $|V(x)| \lesssim(1+|x|)^{-\beta}$ for some $\beta>2$. Let $H=-\triangle+V$ and $\lambda_{1}>0$ be fixed. Then

$$
\sup _{L \geq 1}\left|\left\langle e^{i t H} \chi_{2}(\sqrt{H} / L) \chi_{1}\left(\sqrt{H} / \lambda_{1}\right) f, g\right\rangle\right| \lesssim|t|^{-1}\|f\|_{1}\|g\|_{1}
$$

for all $f, g \in \mathcal{S}\left(\mathbb{R}^{2}\right)$. The constant here depends only on $V$ and $\lambda_{1}$.

Proof. Let $R_{V}^{ \pm}\left(\lambda^{2}\right)=\left(-\triangle+V-\left(\lambda^{2} \pm i 0\right)\right)^{-1}$ be the perturbed resolvent. It satisfies the limiting absorption principle, see Agmon Agm,

$$
\left\|R_{V}^{ \pm}\left(\lambda^{2}\right)\right\|_{L^{2, \sigma}\left(\mathbb{R}^{2}\right) \rightarrow L^{2,-\sigma}\left(\mathbb{R}^{2}\right)}<\infty
$$


provided $\sigma>\frac{1}{2}$. Here $L^{2, \sigma}\left(\mathbb{R}^{2}\right)$ is the usual weighted space with norm

$$
\|f\|_{2, \sigma}=\left(\int_{\mathbb{R}^{2}}(1+|x|)^{2 \sigma}|f(x)|^{2} d x\right)^{\frac{1}{2}} .
$$

In addition, one has

$$
\left\|\partial_{\lambda} R_{V}^{ \pm}\left(\lambda^{2}\right)\right\|_{L^{2, \sigma}\left(\mathbb{R}^{2}\right) \rightarrow L^{2,-\sigma}\left(\mathbb{R}^{2}\right)}<\infty
$$

provided $\sigma>\frac{3}{2}$. The free resolvent satisfies the same bounds with some decay in $\lambda$, say $\lambda^{-\alpha}$. The exact value of $\alpha>0$ is not relevant for our purposes. One has

$$
\begin{aligned}
& \left\langle e^{i t H} \chi_{2}(\sqrt{H} / L) \chi_{1}\left(\sqrt{H} / \lambda_{1}\right) f, g\right\rangle \\
& =\int_{0}^{\infty} e^{i t \lambda^{2}} \lambda \chi_{2}(\lambda / L) \chi_{1}\left(\lambda / \lambda_{1}\right)\left\langle\left[R_{V}^{+}\left(\lambda^{2}\right)-R_{V}^{-}\left(\lambda^{2}\right)\right] f, g\right\rangle \frac{d \lambda}{\pi i} .
\end{aligned}
$$

We use the resolvent expansion

$$
\begin{aligned}
R_{V}^{ \pm}\left(\lambda^{2}\right) & =\sum_{\ell=0}^{2 m+2} R_{0}^{ \pm}\left(\lambda^{2}\right)\left(-V R_{0}^{ \pm}\left(\lambda^{2}\right)\right)^{\ell} \\
& +R_{0}^{ \pm}\left(\lambda^{2}\right)\left(V R_{0}^{ \pm}\left(\lambda^{2}\right)\right)^{m} V R_{V}^{ \pm}\left(\lambda^{2}\right) V\left(R_{0}^{ \pm}\left(\lambda^{2}\right) V\right)^{m} R_{0}^{ \pm}\left(\lambda^{2}\right) .
\end{aligned}
$$

Here $m$ is a positive integer that depends on $\alpha$. Recall that

$$
R_{0}^{ \pm}\left(\lambda^{2}\right)(x, y)= \pm \frac{i}{4} H_{0}^{ \pm}(\lambda|x-y|)
$$

(the Hankel functions of order zero). By Lemma [3] each of the finitely many terms in (18) leads to the desired time-decay in (17). In fact, this only requires that $\|V\|_{\mathcal{K}}<\infty$. For the term (19) one proceeds as in the three-dimensional argument via the limiting absorption principle and stationary phase, see GolSch. Following Yajima Yaj, set

$$
G_{ \pm, x}(\lambda)\left(x_{1}\right):=e^{\mp i \lambda|x|} R_{0}^{ \pm}\left(\lambda^{2}\right)\left(x_{1}, x\right) .
$$

Removing $f, g$ from (17), we are led to proving that

$$
\begin{aligned}
& \mid \int_{0}^{\infty} e^{i t \lambda^{2}} e^{ \pm i \lambda(|x|+|y|)} \chi_{2}(\lambda / L) \chi_{1}\left(\lambda / \lambda_{1}\right) \lambda\left\langle V R_{V}^{ \pm}\left(\lambda^{2}\right) V\left(R_{0}^{ \pm}\left(\lambda^{2}\right) V\right)^{m} G_{ \pm, y}(\lambda),\right. \\
& \left.\quad\left(R_{0}^{\mp}\left(\lambda^{2}\right) V\right)^{m} G_{ \pm, x}^{*}(\lambda)\right\rangle\left. d \lambda|\lesssim| t\right|^{-1}
\end{aligned}
$$

uniformly in $x, y \in \mathbb{R}^{2}$ and $L \geq 1$. Next, we check that the derivatives of $G_{+, x}(\lambda)$ satisfy the estimates (for $\lambda>\lambda_{1}>0$ )

$$
\begin{aligned}
& \sup _{x \in \mathbb{R}^{3}}\left\|\partial_{\lambda}^{j} G_{ \pm, x}(\lambda)\right\|_{L^{2,-\sigma}}<C_{j, \sigma} \lambda^{-\frac{1}{2}}\langle x\rangle^{-\varepsilon} \text { provided } \sigma>\frac{1}{2}+j \\
& \sup _{x \in \mathbb{R}^{3}}\left\|\partial_{\lambda}^{j} G_{ \pm, x}(\lambda)\right\|_{L^{2,-\sigma}}<C_{j, \sigma}(\lambda\langle x\rangle)^{-\frac{1}{2}} \text { provided } \sigma>1+j
\end{aligned}
$$


for all $j \geq 0$. The small $\varepsilon>0$ in (21) depends on $\sigma$. The bound (22) is Lemma 3.1 in Yaj. Alternatively, both bounds follow easily by writing $H_{0}^{ \pm}(u)=e^{ \pm i u} \rho_{ \pm}(u)$ where $\left|\rho_{ \pm}(u)\right| \lesssim\left|\log ^{-}(u)\right| \chi_{\left[0<u<\frac{1}{2}\right]}+u^{-\frac{1}{2}} \chi_{\left[u>\frac{1}{2}\right]}$. Thus, consider

$$
\begin{aligned}
& \left\|\partial_{\lambda}^{j} e^{ \pm i \lambda(|y-x|-|x|)} \rho_{ \pm}(\lambda|x-y|)\langle y\rangle^{-\sigma}\right\|_{L_{y}^{2}\left(\mathbb{R}^{2}\right)}^{2} \\
& \lesssim \int\langle y\rangle^{2(j-\sigma)}\left|\rho_{ \pm}(\lambda|x-y|)\right|^{2} d y \\
& \lesssim \int_{\left[\lambda|x-y|<\frac{1}{2}\right]}\langle y\rangle^{2(j-\sigma)}|\log (\lambda|x-y|)|^{2} d y+\lambda^{-1} \int_{\mathbb{R}^{2}}\langle y\rangle^{2(j-\sigma)}|y-x|^{-1} d y \\
& \lesssim\langle x\rangle^{2(j-\sigma)} \lambda^{-2}+\lambda^{-1}\langle x\rangle^{-1} \chi_{[\sigma>j+1]}+\lambda^{-1}\langle x\rangle^{2(j-\sigma)+1} \chi_{[\sigma<j+1]} .
\end{aligned}
$$

The stated bounds now follow by making the appropriate choices of $\sigma$ depending on $j$.

Rewrite the integral in (20) in the form (with $L=\infty$ )

$$
I^{ \pm}(t, x, y):=\int_{0}^{\infty} e^{i t \lambda^{2} \pm i \lambda(|x|+|y|)} a_{x, y}^{ \pm}(\lambda) d \lambda .
$$

By the aforementioned bounds on $R_{0}^{ \pm}\left(\lambda^{2}\right)$ and $R_{0}^{ \pm}\left(\lambda^{2}\right)$ on weighted $L^{2}$-spaces, which provide decay in $\lambda$, as well as (21), (22), one concludes that $a_{x, y}^{ \pm}(\lambda)$ has one derivative in $\lambda$ and

$$
\begin{aligned}
\left|a_{x, y}^{ \pm}(\lambda)\right| & \lesssim(1+\lambda)^{-2}(\langle x\rangle\langle y\rangle)^{-\frac{1}{2}} \text { for all } \lambda>\lambda_{1} \\
\left|\partial_{\lambda} a_{x, y}^{ \pm}(\lambda)\right| & \lesssim(1+\lambda)^{-2}\langle x\rangle^{-\varepsilon} \quad \text { for all } \lambda>\lambda_{1},
\end{aligned}
$$

which in particular justifies taking $L=\infty$ in (23). This requires that one takes $m$ sufficiently large and that $|V(x)| \lesssim(1+|x|)^{-\beta}$ for some $\beta>2$. The latter condition arises as follows: Consider, (24). Then by (21) and the limiting absorption principle, respectively, we need to write $V=V_{1} V_{2}$, where $V_{1}$ decays like $\langle x\rangle^{-1-\varepsilon}$, whereas the other should decay like $\langle x\rangle^{-\frac{1}{2}-\varepsilon}$. Thus, in this case $\beta>\frac{3}{2}$ is enough. On the other hand, in (25) one derivative may fall on one of the $G$-terms at the ends. Then $V$ has to compensate for a $\frac{3}{2}+\varepsilon$ power because of (21), and also a $\frac{1}{2}+\varepsilon$ power from the limiting absorption principle. Similarly with the other terms.

As far as $I^{+}(t, x, y)$ is concerned, note that on the support of $a_{x, y}^{ \pm}(\lambda)$ the phase $t \lambda^{2}+\lambda(|x|+|y|)$ has no critical point. A single integration by parts yields the bound $\left|I^{+}(t, x, y)\right| \lesssim t^{-1}$ uniformly in $x, y \in \mathbb{R}^{2}$, see (24).

In the case of $I^{-}(t, x, y)$ the phase $t \lambda^{2}-\lambda(|x|+|y|)$ has a unique critical point at $\lambda_{0}=(|x|+|y|) /(2 t)$. If $\lambda_{0} \ll \lambda_{1}$, then a single integration by parts again yields the bound of $t^{-1}$. If $\lambda_{0} \gtrsim \lambda_{1}$ then the bound $\max (|x|,|y|) \gtrsim t$ is also true, and stationary phase contributes $t^{-\frac{1}{2}}(\langle x\rangle\langle y\rangle)^{-\frac{1}{2}} \lesssim t^{-1}$, as desired. To make this 
rigorous, apply Lemma 2

$$
\begin{aligned}
& \left|I^{-}(t, x, y)\right| \\
& \lesssim|t|^{-1} \int \frac{\left|a_{x, y}^{-}(\lambda)\right|}{\delta^{2}+\left|\lambda-\lambda_{0}\right|^{2}} d \lambda+|t|^{-1} \int_{\left[\left|\lambda-\lambda_{0}\right|>\delta\right]} \frac{\left|\partial_{\lambda} a_{x, y}^{-}(\lambda)\right|}{\left|\lambda-\lambda_{0}\right|} d \lambda \\
& \lesssim|t|^{-1} \int_{0}^{\infty} \frac{(1+\lambda)^{-2}(\langle x\rangle\langle y\rangle)^{-\frac{1}{2}}}{\delta^{2}+\left|\lambda-\lambda_{0}\right|^{2}} d \lambda+|t|^{-1} \int_{\left[\left|\lambda-\lambda_{0}\right|>\delta\right]} \frac{(1+\lambda)^{-2}(\langle x\rangle\langle y\rangle)^{-\varepsilon}}{\left|\lambda-\lambda_{0}\right|} d \lambda \\
& \lesssim|t|^{-1}
\end{aligned}
$$

since $\langle x\rangle+\langle y\rangle \gtrsim t$. Note that when $0<t<1$ one has the better bound $\left|I^{ \pm}(t, x, y)\right| \lesssim$ 1 by (24).

\section{Energies close to zero}

The following lemma is a variant of the standard asymptotic expansion around zero energy of the free resolvent on $\mathbb{R}^{2}$. The estimates on the error terms are written in a somewhat unusal form, which is the one needed later in the proof.

Lemma 5. Let $R_{0}^{ \pm}\left(\lambda^{2}\right)=\left(-\triangle-\left(\lambda^{2} \pm i 0\right)\right)^{-1}$ be the free resolvent in $\mathbb{R}^{2}$. Then, for all $\lambda>0$,

$$
R_{0}^{ \pm}\left(\lambda^{2}\right)=\left[ \pm \frac{i}{4}-\frac{1}{2 \pi} \gamma-\frac{1}{2 \pi} \log (\lambda / 2)\right] P_{0}+G_{0}+E_{0}^{ \pm}(\lambda)
$$

Here $P_{0} f:=\int_{\mathbb{R}^{2}} f(x) d x, G_{0} f(x)=-\frac{1}{2 \pi} \int_{\mathbb{R}^{2}} \log |x-y| f(y) d y$, and the error $E_{0}^{ \pm}(\lambda)$ satisfies

$$
\left\|\sup _{0<\lambda} \lambda^{-\frac{1}{2}}\left|E_{0}^{ \pm}(\lambda)(\cdot, \cdot)\right|\right\|+\left\|\sup _{0<\lambda} \lambda^{\frac{1}{2}}\left|\partial_{\lambda} E_{0}^{ \pm}(\lambda)(\cdot, \cdot)\right|\right\| \lesssim 1
$$

with respect to the Hilbert-Schmidt norm in $\mathcal{B}\left(L^{2, s}\left(\mathbb{R}^{2}\right), L^{2,-s}\left(\mathbb{R}^{2}\right)\right)$ with $s>\frac{3}{2}$.

Proof. One has, with $\lambda>0$,

$$
R_{0}^{ \pm}\left(\lambda^{2}\right)(x, y)= \pm \frac{i}{4} H_{0}^{ \pm}(\lambda|x-y|)
$$

where the Hankel functions $H_{0}^{ \pm}$are

$$
\begin{aligned}
H_{0}^{ \pm}(z) & =J_{0}(z) \pm i Y_{0}(z) \\
& =1 \pm i \frac{2}{\pi} \gamma \pm i \frac{2}{\pi} \log (z / 2)+O\left(z^{2} \log z\right), \\
\frac{d}{d z} H_{0}^{ \pm}(z) & = \pm i \frac{2}{\pi z}+O(z \log z) .
\end{aligned}
$$

This is an expansion around $z=0$. Around $z=\infty$ the expansion is given by

$$
H_{0}^{ \pm}(z)=\sqrt{\frac{2}{\pi z}}(a(z) \pm i b(z)) e^{ \pm i(z-\pi / 4)}
$$


with $a(z)=1-\frac{\alpha}{z^{2}} \pm \ldots$ and $b(z)=\frac{\beta}{z} \pm \ldots$. Now let

$$
E_{0}^{ \pm}(\lambda)(x, y):=R_{0}^{ \pm}\left(\lambda^{2}\right)(x, y)-\left[ \pm \frac{i}{4}-\frac{1}{2 \pi} \gamma-\frac{1}{2 \pi} \log (\lambda / 2)\right]+\frac{1}{2 \pi} \log |x-y| .
$$

Then

$$
\begin{aligned}
& \left|E_{0}^{ \pm}(\lambda)(x, y)\right| \\
& \lesssim \lambda^{2}|x-y|^{2}|\log (\lambda|x-y|)| \chi_{[\lambda|x-y| \leq 1]}+[1+\log (\lambda|x-y|)] \chi_{[\lambda|x-y|>1]} \\
& \lesssim \lambda^{2 \varepsilon}|x-y|^{2 \varepsilon}|\log (\lambda|x-y|)| \chi_{[\lambda|x-y| \leq 1]}+[1+\log (\lambda|x-y|)] \chi_{[\lambda|x-y|>1]} .
\end{aligned}
$$

Hence

$$
\sup _{0<\lambda} \lambda^{-\varepsilon}\left|E_{0}^{ \pm}(\lambda)(x, y)\right| \lesssim|x-y|^{\varepsilon} .
$$

Since the right-hand side has finite Hilbert-Schmidt norm as an operator $L^{2, s}\left(\mathbb{R}^{2}\right) \rightarrow$ $L^{2,-s}\left(\mathbb{R}^{2}\right)$ ) with $s>1+\varepsilon$, we obtain the first part of (27). On the other hand,

$$
\begin{aligned}
& \lambda^{1-\varepsilon}\left|\partial_{\lambda} E_{0}^{ \pm}(\lambda)(x, y)\right| \\
& \lesssim \lambda^{2-\varepsilon}|x-y|^{2}|\log (\lambda|x-y|)| \chi_{[\lambda|x-y| \leq 1]}+\left[\lambda^{\frac{1}{2}-\varepsilon}|x-y|^{\frac{1}{2}}+\lambda^{-\varepsilon}\right] \chi_{[\lambda|x-y|>1]} \\
& \lesssim|x-y|^{\varepsilon}+\lambda^{\frac{1}{2}-\varepsilon}|x-y|^{\frac{1}{2}} \chi_{[\lambda|x-y|>1]},
\end{aligned}
$$

and therefore, setting $\varepsilon=\frac{1}{2}$,

$$
\sup _{0<\lambda} \lambda^{\frac{1}{2}}\left|\partial_{\lambda} E_{0}^{ \pm}(\lambda)(x, y)\right| \lesssim|x-y|^{\frac{1}{2}}
$$

Since the right-hand side has finite Hilbert-Schmidt norm as an operator $L^{2, s}\left(\mathbb{R}^{2}\right) \rightarrow$ $L^{2,-s}\left(\mathbb{R}^{2}\right)$ with $s>\frac{3}{2}$, the lemma follows.

Now let $V: \mathbb{R}^{2} \rightarrow \mathbb{R}, V \not \equiv 0$, satisfy $|V(x)| \lesssim(1+|x|)^{-2 \beta}$ for $\beta>\frac{3}{2}$ (this condition arises because of the condition $s>\frac{3}{2}$ in the previous lemma). Following Jensen and Nenciu [JenNen] we set $U(x)=1$ if $V(x) \geq 0$ and $U(x)=-1$ if $V(x)<0$. Also, $v(x):=|V(x)|^{\frac{1}{2}}$ decays like $(1+|x|)^{-\beta}$. The following corollary is therefore an immediate consequence of Lemma 5 .

Corollary 6. For $\lambda>0$ define $M^{ \pm}(\lambda):=U+v R_{0}^{ \pm}\left(\lambda^{2}\right) v$. Let $P=\frac{v\langle\cdot, v\rangle}{\|V\|_{1}}$ denote the orthogonal projection onto $v$. Then

$$
M^{ \pm}(\lambda)=g^{ \pm}(\lambda) P+U+v G_{0} v+v E_{0}^{ \pm}(\lambda) v
$$

Here $G_{0}, E_{0}^{ \pm}(\lambda)$ are as in Lemma 5 and $g^{ \pm}(\lambda)=\|V\|_{1}\left( \pm \frac{i}{4}-\frac{1}{2 \pi} \gamma-\frac{1}{2 \pi} \log (\lambda / 2)\right)$. The remainders satisfy

$$
\left\|v \sup _{0<\lambda} \lambda^{-\frac{1}{2}}\left|E_{0}^{ \pm}(\lambda)(\cdot, \cdot)\right| v\right\|_{H S}+\left\|v \sup _{0<\lambda} \lambda^{\frac{1}{2}}\left|\partial_{\lambda} E_{0}^{ \pm}(\lambda)(\cdot, \cdot)\right| v\right\|_{H S} \lesssim 1
$$

with respect to the Hilbert-Schmidt norm on $L^{2}\left(\mathbb{R}^{2}\right)$. 
The following definition is motivated by JenNen], cf. the case of $S_{1}=0$ in their Theorem 6.2.

Definition 7. Let $Q=1-P$. We say that zero is a regular point of the spectrum of $H=-\triangle+V$ provided $Q\left(U+v G_{0} v\right) Q$ is invertible on $Q L^{2}\left(\mathbb{R}^{2}\right)$. In that case set $D_{0}:=\left[Q\left(U+v G_{0} v\right) Q\right]^{-1}$ as an operator on $Q L^{2}\left(\mathbb{R}^{2}\right)$.

Jensen and Nenciu show that $Q\left(U+v G_{0} v\right) \Phi=0$ with $\Phi \in Q L^{2}\left(\mathbb{R}^{2}\right)$ implies that $\Phi=$ $U v \Psi$ where $H \Psi=0$ in the sense of distributions and $\Psi \in L^{\infty}\left(\mathbb{R}^{2}\right)$. Thus Definition[7 captures what is sometimes described as absence of zero-energy eigenfunctions and resonances.

The following lemma is a technical statement that will be used repeatedly in our argument.

Lemma 8. Let $D_{0}$ be as in Definition 7. Let $K$ be the kernel of the operator $Q D_{0} Q$. Then the operator with kernel $|K|$ is again $L^{2}$-bounded.

Proof. For the purposes of this proof we introduce the following terminology: A bounded operator $T$ on $L^{2}\left(\mathbb{R}^{2}\right)$ is called absolutely bounded if the absolute value of its kernel gives rise to a bounded operator on $L^{2}\left(\mathbb{R}^{2}\right)$. Note that Hilbert-Schmidt operators are absolutely bounded.

Suppose $f \in Q L^{2}\left(\mathbb{R}^{2}\right)$ such that $Q U f=0, f \neq 0$. Then $U f=c v$ for some scalar $c \neq$ 0 . Hence $f=c U v$ and $P f=0$ requires that $\langle f, v\rangle=c\langle U v, v\rangle=c \int_{\mathbb{R}^{2}} V(x) d x=0$. Since this argument can be reversed,

$$
\operatorname{ker}_{Q L^{2}}(Q U Q)=\{0\} \quad \text { iff } \int_{\mathbb{R}^{2}} V(x) d x \neq 0
$$

Case 1: $\int_{\mathbb{R}^{2}} V(x) d x \neq 0$.

In this case we claim that $Q U Q: Q L^{2}\left(\mathbb{R}^{2}\right) \rightarrow Q L^{2}\left(\mathbb{R}^{2}\right)$ is invertible. More precisely, one checks that for any $g \in L^{2}$ with $Q g=g$

$$
f=U g+c_{0} U v \text { with } c_{0}=-\frac{\langle U g, v\rangle}{\int_{\mathbb{R}^{2}} V(x) d x}
$$

solves $Q U Q f=g, Q f=f$. It is evident from this explicit formula that $Q(Q U Q)^{-1} Q$ is absolutely bounded. Moreover, on $Q L^{2}$,

$$
\left[Q\left(U+v G_{0} v\right) Q\right]^{-1}=(Q U Q)^{-1}\left[Q+Q v G_{0} v(Q U Q)^{-1} Q\right]^{-1} .
$$

Now $v G_{0} v$ is a Hilbert-Schmidt operator since $v$ decays faster than $(1+|x|)^{-1-\varepsilon}$. Hence $W:=Q v G_{0} v(Q U Q)^{-1} Q$ is also Hilbert-Schmidt. Finally, as an identity on $Q L^{2}$

$$
\left[Q+Q v G_{0} v(Q U Q)^{-1} Q\right]^{-1}-Q=-[Q+W]^{-1} W .
$$

Since the right-hand side is Hilbert-Schmidt, we see from (31) that $\left[Q\left(U+v G_{0} v\right) Q\right]^{-1}$ is the composition of an absolutely bounded operator with the sum of an absolutely 
bounded operator and a Hilbert-Schmidt operator. Hence it is itself absolutely bounded, as claimed.

Case 2: $\int_{\mathbb{R}^{2}} V(x) d x=0$.

In this case we remark that 0 is an isolated point of the spectrum of $Q U Q$. Let $\pi_{0}$ denote the Riesz projection onto $\operatorname{ker}(Q U Q)$ in $Q L^{2}$. From the preceding, $\pi_{0}(f)=\|V\|_{1}^{-1}\langle f, U v\rangle U v$. Then $Q U Q+\pi_{0}$ is invertible on $Q L^{2}\left(\mathbb{R}^{2}\right)$. In fact, one checks that an explicit solution of

$$
\left(Q U Q+\pi_{0}\right) f=g \text { where } Q g=g, Q f=f
$$

is given by

$$
f=U g+c_{1} v-c_{1} U v \text { with } c_{1}=-\frac{\langle g, U v\rangle}{\int_{\mathbb{R}^{2}}|V(x)| d x} .
$$

In view of this explicit expression, $\left(Q U Q+\pi_{0}\right)^{-1}$ is absolutely bounded on $Q L^{2}$. Finally, the identity

$$
\left[Q\left(U+v G_{0} v\right) Q\right]^{-1}=\left[Q U Q+\pi_{0}+Q v G_{0} v Q-\pi_{0}\right]^{-1}
$$

on $Q L^{2}$ allows one to repeat the same argument as in Case 1 and the lemma follows.

The main technical result in Jensen and Nenciu JenNen is a formula for the inverse of $M^{ \pm}(\lambda)^{-1}$. In the general case, this is complicated, see their Theorem 6.2. But since we are imposing the condition of Definition [7, it is relatively simple to compute that inverse, see the following lemma. Since we need somewhat stronger bounds on the error than those obtained in JenNen, we give all details. In particular, the proof requires Lemma 8

Lemma 9. Suppose that zero is a regular point of the spectrum of $H=-\triangle+V$. Then for some sufficiently small $\lambda_{1}>0$, the operators $M^{ \pm}(\lambda)$ are invertible for all $0<\lambda<\lambda_{1}$ as bounded operators on $L^{2}\left(\mathbb{R}^{2}\right)$, and one has the expansion

$$
M^{ \pm}(\lambda)^{-1}=h_{ \pm}(\lambda)^{-1} S+Q D_{0} Q+E^{ \pm}(\lambda)
$$

where $h_{+}(\lambda)=a \log \lambda+z$, a is real, $z$ complex, $a \neq 0, \Im z \neq 0$, and $h_{-}(\lambda)=\overline{h_{+}(\lambda)}$. Moreover, $S$ is of finite rank and has a real-valued kernel, and $E^{ \pm}(\lambda)$ is a HilbertSchmidt operator that satisfies the bound

$$
\left\|\sup _{0<\lambda<\lambda_{1}} \lambda^{-\frac{1}{2}}\left|E^{ \pm}(\lambda)(\cdot, \cdot)\right|\right\|_{H S}+\left\|\sup _{0<\lambda<\lambda_{1}} \lambda^{\frac{1}{2}}\left|\partial_{\lambda} E^{ \pm}(\lambda)(\cdot, \cdot)\right|\right\|_{H S} \lesssim 1
$$

where the norm refers to the Hilbert-Schmidt norm on $L^{2}\left(\mathbb{R}^{2}\right)$. Finally, let $R_{V}^{ \pm}\left(\lambda^{2}\right)=$ $\left(-\triangle+V-\left(\lambda^{2} \pm i 0\right)\right)^{-1}$. Then

$$
R_{V}^{ \pm}\left(\lambda^{2}\right)=R_{0}^{ \pm}\left(\lambda^{2}\right)-R_{0}^{ \pm}\left(\lambda^{2}\right) v M^{ \pm}(\lambda)^{-1} v R_{0}^{ \pm}\left(\lambda^{2}\right) .
$$

This is to be understood as an identity between operators $L^{2, \frac{1}{2}+\varepsilon}\left(\mathbb{R}^{2}\right) \rightarrow L^{2,-\frac{1}{2}-\varepsilon}\left(\mathbb{R}^{2}\right)$ for some sufficiently small $\varepsilon>0$. 
Proof. For the purposes of this proof set $T=U+v G_{0} v$. By assumption, $Q T Q$ is invertible on $Q L^{2}\left(\mathbb{R}^{2}\right)$. Moreover, by Corollary [6. with respect to the decomposition $L^{2}\left(\mathbb{R}^{2}\right)=P L^{2}\left(\mathbb{R}^{2}\right) \oplus Q L^{2}\left(\mathbb{R}^{2}\right)$,

$$
M^{ \pm}(\lambda)=\left[\begin{array}{cc}
g^{ \pm}(\lambda) P+P T P & P T Q \\
Q T P & Q T Q
\end{array}\right]+v E_{0}^{ \pm}(\lambda) v
$$

Denote the matrix on the right-hand side by $A(\lambda)=\left[\begin{array}{ll}a_{11} & a_{12} \\ a_{21} & a_{22}\end{array}\right]$. To invert $M^{ \pm}(\lambda)$ and thus $A(\lambda)$, we use the well-known Fehsbach formula, see eg. Lemma 2.3 in [JenNen]. This requires that $a:=\left(a_{11}-a_{12} a_{22}^{-1} a_{21}\right)^{-1}$ exists, and in that case

$$
A(\lambda)^{-1}=\left[\begin{array}{cc}
a & -a a_{12} a_{22}^{-1} \\
-a_{22}^{-1} a_{21} a & a_{22}^{-1} a_{21} a a_{12} a_{22}^{-1}+a_{22}^{-1}
\end{array}\right]
$$

Note that in our case, as an operator on the line $\operatorname{Ran}(P)=\{c v: c \in \mathbb{C}\}$,

$$
a=h_{ \pm}(\lambda)^{-1} P \text { where } h_{ \pm}(\lambda):=g^{ \pm}(\lambda)+\operatorname{trace}\left(P T P-P T Q D_{0} Q T P\right) .
$$

The trace is real-valued since $v$ is real-valued and since the kernel of $T$ is real-valued. In view of the definition of $g^{ \pm}(\lambda), h_{ \pm}(\lambda) \neq 0$ provided $\lambda>0$ is sufficiently small. Moreover, by (35) we see that

$$
A(\lambda)^{-1}=h_{ \pm}(\lambda)^{-1} S+Q D_{0} Q
$$

where $S$ is of finite rank (in fact, the rank is at most two). By the definition of $h_{ \pm}(\lambda)$ and by Lemma 8

$$
\left|A(\lambda)^{-1}(\cdot, \cdot)\right|+\lambda\left|\partial_{\lambda} A(\lambda)^{-1}(\cdot, \cdot)\right| \lesssim|S(\cdot, \cdot)|+\left|Q D_{0} Q(\cdot, \cdot)\right|,
$$

where the right-hand side is an $L^{2}$-bounded operator. Now

$$
M^{ \pm}(\lambda)^{-1}=A(\lambda)^{-1}\left[1+v E_{0}^{ \pm}(\lambda) v A(\lambda)^{-1}\right]^{-1} .
$$

The second inverse on the right-hand side exists for small $\lambda$ since then

$$
\left\|v E_{0}^{ \pm}(\lambda) v A(\lambda)^{-1}\right\|<\frac{1}{2}
$$

see (301). Moreover, writing out $E^{ \pm}(\lambda)$ as a Neuman series, one obtains (33) from (30) and (36) by termwise estimation.

Finally, (34) is the well-known symmetric resolvent expansion which follows easily from

$$
\begin{aligned}
& \left(I-U v(-\triangle+V-z)^{-1} v\right)\left(I+U v(-\triangle-z)^{-1} v\right)=I \quad \text { or } \\
& V(-\triangle+V-z)^{-1} V=V-v\left(U+v(-\triangle-z)^{-1} v\right)^{-1} v
\end{aligned}
$$

for $\Im z>0$. Passing to the limit $\Im z \rightarrow 0$ now leads to (34) via an application of the resolvent identity and the limiting absorption principle, cf. (16). 
Corollary 10. Let zero be a regular point of the spectrum of $H=-\triangle+V$. Then

$$
\begin{aligned}
R_{V}^{ \pm}\left(\lambda^{2}\right) & =R_{0}^{ \pm}\left(\lambda^{2}\right)-h_{ \pm}(\lambda)^{-1} R_{0}^{ \pm}\left(\lambda^{2}\right) v S v R_{0}^{ \pm}\left(\lambda^{2}\right) \\
& -R_{0}^{ \pm}\left(\lambda^{2}\right) v Q D_{0} Q v R_{0}^{ \pm}\left(\lambda^{2}\right)-R_{0}^{ \pm}\left(\lambda^{2}\right) v E^{ \pm}(\lambda) v R_{0}^{ \pm}\left(\lambda^{2}\right)
\end{aligned}
$$

where $S$ and $E^{ \pm}(\lambda)$ are as in the previous lemma. This is to be understood as an identity between operators $L^{2, \frac{1}{2}+\varepsilon}\left(\mathbb{R}^{2}\right) \rightarrow L^{2,-\frac{1}{2}-\varepsilon}\left(\mathbb{R}^{2}\right)$ for small $\varepsilon>0$, i.e., as in the limiting absorption principle (16).

Proof. This is an immediate consequence of Corollary [6] and Lemma 9 .

We now turn to decay estimates.

Proposition 11. Let $\chi$ be a smooth cut-off function on the line with $\chi(\lambda)=1$ if $\lambda \leq \lambda_{1}$ and $\chi(\lambda)=0$ if $\lambda \geq 2 \lambda_{1}$ where $\lambda_{1}>0$ is a small constant. Assume that zero is a regular point of the spectrum of $H=-\triangle+V$. Then

$$
\begin{aligned}
\left|\left\langle e^{i t H} \chi(\sqrt{H}) P_{a c}(H) f, g\right\rangle\right| & =\frac{1}{\pi}\left|\int_{0}^{\infty} e^{i t \lambda^{2}} \lambda \chi(\lambda)\left\langle\left[R_{V}^{+}\left(\lambda^{2}\right)-R_{V}^{-}\left(\lambda^{2}\right)\right] f, g\right\rangle d \lambda\right| \\
& \leq C|t|^{-1}\|f\|_{1}\|g\|_{1}
\end{aligned}
$$

for every $f, g \in \mathcal{S}\left(\mathbb{R}^{2}\right)$. Here $C$ is a constant that only depends on $V$ and $\chi$.

The proof of Proposition [1] is based on the expansion of $R_{V}^{ \pm}\left(\lambda^{2}\right)$ stated in Corollary 10. Each of the four terms on the right-hand side of (37) requires a separate argument. We begin with the free case.

Lemma 12. $H_{0}=-\triangle$ satisfies

$$
\left|\left\langle e^{i t H_{0}} \chi\left(\sqrt{H_{0}}\right) P_{a c}(H) f, g\right\rangle\right| \leq C|t|^{-1}\|f\|_{1}\|g\|_{1}
$$

for all $f, g \in \mathcal{S}\left(\mathbb{R}^{2}\right)$.

Proof. This follows immediately from the standard bound $\left\|e^{i t H_{0}} f\right\|_{\infty} \lesssim|t|^{-1}\|f\|_{1}$ and the fact that $\chi\left(\sqrt{H_{0}}\right)$ and $P_{a c}(H)$ are bounded on $L^{1}\left(\mathbb{R}^{2}\right)$ (for the latter, use that the number of negative bound states is finite Sto, as well as that the eigenfunctions are exponentially decaying by Agmon's bound, and therefore in $L^{1}\left(\mathbb{R}^{2}\right)$. Moreover, they are in $L^{\infty}\left(\mathbb{R}^{2}\right)$ by Sobolev imbedding). Alternatively, one can give a selfcontained proof via stationary phase. Indeed, from (28)

$$
R_{0}^{+}\left(\lambda^{2}\right)(x, y)-R_{0}^{-}\left(\lambda^{2}\right)(x, y)=\frac{i}{2} J_{0}(\lambda|x-y|) .
$$

Thus

$$
\begin{aligned}
& \left|\left\langle e^{i t H_{0}} \chi\left(\sqrt{H_{0}}\right) P_{a c}(H) f, g\right\rangle\right| \\
& \leq \int_{\mathbb{R}^{4}} \frac{1}{2 \pi}\left|\int_{0}^{\infty} e^{i t \lambda^{2}} \lambda \chi(\lambda) J_{0}(\lambda|x-y|) d \lambda\right|\left|P_{a c}(H) f(x)\right||g(y)| d x d y .
\end{aligned}
$$


Now $J_{0}(u)=e^{i u} \omega_{+}(u)+e^{-i u} \omega_{-}(u)$ where $\left|\omega_{ \pm}(u)\right| \lesssim(1+|u|)^{-\frac{1}{2}}$. Therefore,

$$
\begin{aligned}
& \left|\int_{0}^{\infty} e^{i t \lambda^{2}} \lambda \chi(\lambda) J_{0}(\lambda|x-y|) d \lambda\right| \\
& \lesssim\left|\int_{0}^{\infty} e^{i\left[t \lambda^{2}-\lambda|x-y|\right]} \lambda \chi(\lambda) \omega_{+}(\lambda|x-y|) d \lambda\right| \\
& +\left|\int_{0}^{\infty} e^{i\left[t \lambda^{2}+\lambda|x-y|\right]} \lambda \chi(\lambda) \omega_{-}(\lambda|x-y|) d \lambda\right| .
\end{aligned}
$$

Let $t>0$. The phase in (39) has a stationary point $\lambda_{0}=\frac{|x-y|}{2 t}$. Hence that integral is

$$
\lesssim t^{-\frac{1}{2}} \lambda_{0}\left(1+\lambda_{0}^{2} t\right)^{-\frac{1}{2}} \lesssim t^{-1}
$$

by stationary phase (we leave it to the reader to fill in the remaining details here). The integral in (40) can be estimated directly by means of integration by parts.

The following lemmas deal with the contribution of the term containing $Q D_{0} Q$ in (37). In what follows it will be assumed that zero is a regular point of the spectrum of $H=-\triangle+V$.

Lemma 13. Let $\left(Q D_{0} Q\right)(\cdot, \cdot)$ denote the kernel of $Q D_{0} Q$. There is the bound

$$
\begin{aligned}
& \mid \int_{\mathbb{R}^{8}} \int_{0}^{\infty} e^{i t \lambda^{2}} \lambda \chi(\lambda) \chi\left(\lambda\left|x-x_{1}\right|\right) Y_{0}\left(\lambda\left|x-x_{1}\right|\right) v\left(x_{1}\right)\left(Q D_{0} Q\right)\left(x_{1}, y_{1}\right) v\left(y_{1}\right) \\
& \left.J_{0}\left(\lambda\left|y_{1}-y\right|\right) \chi\left(\lambda\left|y_{1}-y\right|\right) d \lambda f(x) g(y) d x_{1} d y_{1} d x d y|\leq C| t\right|^{-1}\|f\|_{1}\|g\|_{1}
\end{aligned}
$$

with a constant that only depends on $V$.

Proof. We make the following claim:

$$
\begin{aligned}
& \mid \int_{0}^{\infty} e^{i t \lambda^{2}} \lambda \chi(\lambda)\left[\chi\left(\lambda\left|x-x_{1}\right|\right) Y_{0}\left(\lambda\left|x-x_{1}\right|\right)-\frac{2}{\pi} \chi(\lambda(1+|x|)) \log (\lambda(1+|x|))\right] \\
& \left.J_{0}\left(\lambda\left|y_{1}-y\right|\right) \chi\left(\lambda\left|y_{1}-y\right|\right) d \lambda|\leq C| t\right|^{-1}\left(1+\log ^{+}\left|x_{1}\right|+\log ^{-}\left|x-x_{1}\right|\right)
\end{aligned}
$$

for all $x, x_{1}, y, y_{1} \in \mathbb{R}^{2}$. Let

$$
k\left(x, x_{1}\right):=1+\log ^{+}\left|x_{1}\right|+\log ^{-}\left|x-x_{1}\right| .
$$

If (42) holds, then the left-hand side of (41) is

$$
\begin{aligned}
& \lesssim|t|^{-1} \int_{\mathbb{R}^{8}} k\left(x, x_{1}\right) v\left(x_{1}\right)\left|\left(Q D_{0} Q\right)\left(x_{1}, y_{1}\right)\right| v\left(y_{1}\right)|f(x) \| g(y)| d x_{1} d y_{1} d x d y \\
& \lesssim|t|^{-1} \sup _{x \in \mathbb{R}^{2}}\left(\int_{\mathbb{R}^{2}} k\left(x, x_{1}\right)^{2}|V|\left(x_{1}\right) d x_{1}\right)^{\frac{1}{2}}\left\|\left|Q D_{0} Q\right|\right\|_{2 \rightarrow 2}\|V\|_{1}^{\frac{1}{2}}\|f\|_{1}\|g\|_{1} \\
& \lesssim|t|^{-1}\|f\|_{1}\|g\|_{1},
\end{aligned}
$$


as desired. To see this, observe firstly that

$$
\int_{\mathbb{R}^{4}} v(x)\left(Q D_{0} Q\right)(x, y) h(y) d x d y=0
$$

for any $h \in L^{2}\left(\mathbb{R}^{2}\right)$. Secondly, use Lemma 8 to control the $L^{2}$-operator norm of the kernel $\left|Q D_{0} Q\right|$. To prove (42), let

$$
\begin{aligned}
& F\left(\lambda, x, x_{1}\right):=\chi\left(\lambda\left|x-x_{1}\right|\right) Y_{0}\left(\lambda\left|x-x_{1}\right|\right)-\frac{2}{\pi} \chi(\lambda(1+|x|)) \log (\lambda(1+|x|)) \\
& G\left(\lambda, y_{1}, y\right):=J_{0}\left(\lambda\left|y_{1}-y\right|\right) \chi\left(\lambda\left|y_{1}-y\right|\right) .
\end{aligned}
$$

If we choose $1>\lambda_{1}>0$ so that $2 \lambda_{1}$ lies to the left of the first zero of $J_{0}$, then $G\left(\lambda, y_{1}, y\right)$ is nonincreasing in $\lambda$ (recall the definition of $\chi$ in Proposition 11). Moreover, in that case $0 \leq G \leq 1$ for all choices of arguments. Recall that $J_{0}(z)=1+O\left(z^{2}\right)$ and

$$
\begin{aligned}
& Y_{0}(z)=\frac{2}{\pi}(\log z+c) J_{0}(z)+r(z) \\
& Y_{0}^{\prime}(z)=\frac{2}{\pi z} J_{0}(z)+\frac{2}{\pi}(\log z+c) J_{0}^{\prime}(z)+r^{\prime}(z)=\frac{2}{\pi z}+g(z)
\end{aligned}
$$

where $r(z)$ is analytic for all $z$ and $g(z)$ bounded on $(0, \infty)$, say. Hence one has $F\left(0+, x, x_{1}\right)=\frac{2}{\pi} c+\frac{2}{\pi} \log \frac{\left|x-x_{1}\right|}{1+|x|}$, and $G\left(0, y_{1}, y\right)=1$. It is easy to check that

$$
\left|\log \frac{\left|x-x_{1}\right|}{1+|x|}\right| \lesssim 1+\log ^{+}\left|x_{1}\right|+\log ^{-}\left|x-x_{1}\right|=k\left(x, x_{1}\right)
$$

Indeed, if $|x| \geq 2\left|x_{1}\right|$, then

$$
\frac{1}{4} \chi_{[|x| \geq 1]}+\frac{1}{2}\left|x-x_{1}\right| \chi_{[|x| \leq 1]} \leq \frac{\left|x-x_{1}\right|}{1+|x|} \leq \frac{2|x|}{1+|x|} \leq 2 .
$$

On the other hand, if $|x|<2\left|x_{1}\right|$, then

$$
\frac{\min \left(1,\left|x-x_{1}\right|\right)}{1+2\left|x_{1}\right|} \leq \frac{\left|x-x_{1}\right|}{1+|x|} \leq \frac{3\left|x_{1}\right|}{1+|x|} \leq 3\left|x_{1}\right|,
$$

and (49) follows. Integrating by parts inside the integral in (42) therefore leads to the estimate

$$
\begin{aligned}
\text { (42) } \lesssim & |t|^{-1} k\left(x, x_{1}\right)+|t|^{-1} \int_{0}^{\infty}\left|\chi^{\prime}(\lambda)\right|\left|F\left(\lambda, x, x_{1}\right)\right|\left|G\left(\lambda, y_{1}, y\right)\right| d \lambda \\
& +|t|^{-1} \int_{0}^{\infty}\left|\partial_{\lambda} F\left(\lambda, x, x_{1}\right)\right|\left|G\left(\lambda, y_{1}, y\right)\right| d \lambda \\
& +|t|^{-1} \int_{0}^{\infty}\left|F\left(\lambda, x, x_{1}\right)\right|\left|\partial_{\lambda} G\left(\lambda, y_{1}, y\right)\right| d \lambda .
\end{aligned}
$$


Recall that the support of $\chi^{\prime}$ is contained inside $\left[\lambda_{1}, 2 \lambda_{1}\right]$. Thus the integral involving $\chi^{\prime}(\lambda)$ is easily seen to be

$$
\lesssim \sup _{\lambda \sim \lambda_{1}}\left|F\left(\lambda, x, x_{1}\right)\right|\left|G\left(\lambda, y_{1}, y\right)\right| \lesssim 1+\log ^{-}\left|x-x_{1}\right|,
$$

cf. (47). With the notation of (48),

$$
\begin{aligned}
& \partial_{\lambda} F\left(\lambda, x, x_{1}\right)=\frac{2}{\pi} \frac{1}{\lambda}\left[\chi\left(\lambda\left|x-x_{1}\right|\right)-\chi(\lambda(1+|x|))\right] \\
& +\left|x-x_{1}\right| \chi^{\prime}\left(\lambda\left|x-x_{1}\right|\right) Y_{0}\left(\lambda\left|x-x_{1}\right|\right)+\left|x-x_{1}\right| \chi\left(\lambda\left|x-x_{1}\right|\right) g\left(\lambda\left|x-x_{1}\right|\right) \\
& -\frac{2}{\pi} \chi^{\prime}(\lambda(1+|x|))(1+|x|) \log (\lambda(1+|x|)) .
\end{aligned}
$$

Hence,

$$
\begin{aligned}
\text { (501) } & \lesssim|t|^{-1} \int_{0}^{\infty}\left|\chi\left(\lambda\left|x-x_{1}\right|\right)-\chi(\lambda(1+|x|))\right| \lambda^{-1} d \lambda \\
& +|t|^{-1} \int_{0}^{\infty}\left|x-x_{1}\right|\left[\left|\chi^{\prime}\left(\lambda\left|x-x_{1}\right|\right)\right|+\chi\left(\lambda\left|x-x_{1}\right|\right)\right] d \lambda \\
& +|t|^{-1} \int_{0}^{\infty}(1+|x|)\left|\chi^{\prime}(\lambda(1+|x|))\right| d \lambda \\
& \lesssim|t|^{-1}\left[1+\log ^{+}\left(\frac{2\left|x-x_{1}\right|}{1+|x|}\right)+\log ^{+}\left(\frac{2(1+|x|)}{\left|x-x_{1}\right|}\right)\right] \lesssim|t|^{-1} k\left(x, x_{1}\right)
\end{aligned}
$$

where we used (49) in the last step. In passing, we note that we have shown the following:

$$
\sup _{0 \leq \lambda \leq 1}\left|F\left(\lambda, x, x_{1}\right)\right| \leq\left|F\left(0, x, x_{1}\right)\right|+\int_{0}^{1}\left|\partial_{\lambda} F\left(\lambda, x, x_{1}\right)\right| d \lambda \lesssim k\left(x, x_{1}\right) .
$$

As observed previously, $\partial_{\lambda} G$ has a definite sign. Moreover, $F\left(\lambda, x, x_{1}\right)$ only has a finite number of zeros in $\lambda$. Hence, one can break up the integral (51) into finitely many disjoint intervals, remove the absolute values on each of them, and then integrate by parts. The only boundary contribution occurs at $\lambda=0$, for which we have already obtained the desired bound. Otherwise, the remaining integral is bounded above by (50), and we are done.

The following lemma deals with an integral very much like the one in (41). The difference here is that we consider the contribution from large arguments inside $J_{0}$, which makes it necessary to exploit the oscillations of $J_{0}$. This will be done by means of Lemma 2

Lemma 14. Let $\left(Q D_{0} Q\right)(\cdot, \cdot)$ denote the kernel of $Q D_{0} Q$. Let $\tilde{\chi}=1-\chi$. Then there is the bound

$$
\begin{aligned}
& \mid \int_{\mathbb{R}^{8}} \int_{0}^{\infty} e^{i t \lambda^{2}} \lambda \chi(\lambda) \chi\left(\lambda\left|x-x_{1}\right|\right) Y_{0}\left(\lambda\left|x-x_{1}\right|\right) v\left(x_{1}\right)\left(Q D_{0} Q\right)\left(x_{1}, y_{1}\right) v\left(y_{1}\right) \\
& \left.J_{0}\left(\lambda\left|y_{1}-y\right|\right) \tilde{\chi}\left(\lambda\left|y_{1}-y\right|\right) d \lambda f(x) g(y) d x_{1} d y_{1} d x d y|\leq C| t\right|^{-1}\|f\|_{1}\|g\|_{1}
\end{aligned}
$$


with a constant that only depends on $V$. The same statement holds with the role of the cut-offs interchanged, i.e., with $\tilde{\chi}\left(\lambda\left|x-x_{1}\right|\right)$ and $\chi\left(\lambda\left|y-y_{1}\right|\right)$.

Proof. As usual,

$$
J_{0}(y)=e^{i y} \omega_{+}(y)+e^{-i y} \omega_{-}(y)
$$

where $\left|\omega_{ \pm}^{(\ell)}(y)\right| \lesssim(1+|y|)^{-\frac{1}{2}-\ell}$ for all $\ell \geq 0$. Correspondingly, there will be two contributions to (54). We start with the phase $\phi_{-}(\lambda)=\lambda^{2}-\lambda\left|y-y_{1}\right| t^{-1}$ which has a critical point at $\lambda_{0}=\frac{\left|y-y_{1}\right|}{2 t}$. In that case we claim that

$$
\begin{aligned}
& \mid \int_{0}^{\infty} e^{i t \phi_{-}(\lambda)} \lambda \chi(\lambda)\left[\chi\left(\lambda\left|x-x_{1}\right|\right) Y_{0}\left(\lambda\left|x-x_{1}\right|\right)-\frac{2}{\pi} \chi(\lambda(1+|x|)) \log (\lambda(1+|x|))\right] \\
& \left.\omega_{-}\left(\lambda\left|y_{1}-y\right|\right) \tilde{\chi}\left(\lambda\left|y_{1}-y\right|\right) d \lambda|\leq C| t\right|^{-1} k\left(x, x_{1}\right)
\end{aligned}
$$

for all $x, x_{1}, y, y_{1} \in \mathbb{R}^{2}$. Here $k\left(x, x_{1}\right)$ is as in (43). Moreover, as in the previous proof, this bound will lead to the desired estimate in (54) in view of (44). With $F\left(\lambda, x, x_{1}\right)$ as in (45), set

$$
a(\lambda):=\lambda \chi(\lambda) \omega_{-}\left(\lambda\left|y-y_{1}\right|\right) \tilde{\chi}\left(\lambda\left|y-y_{1}\right|\right) F\left(\lambda, x, x_{1}\right)
$$

where we suppress the other variables inside $a$. By Lemma 2

$$
\left|\int_{0}^{\infty} e^{i t \phi_{-}(\lambda)} a(\lambda) d \lambda\right| \lesssim|t|^{-1} \int_{-\infty}^{\infty}\left(\frac{|a(\lambda)|}{\delta^{2}+\left|\lambda-\lambda_{0}\right|^{2}}+\frac{\left|a^{\prime}(\lambda)\right|}{\left|\lambda-\lambda_{0}\right|} \chi_{\left[\left|\lambda-\lambda_{0}\right|>\delta\right]}\right) d \lambda .
$$

To establish our claim we therefore need to show that the integral in (58) is $\lesssim$ $k\left(x, x_{1}\right)$. Using (53) one concludes

$$
\begin{aligned}
& \int_{-\infty}^{\infty} \frac{|a(\lambda)|}{\delta^{2}+\left|\lambda-\lambda_{0}\right|^{2}} d \lambda \\
& \lesssim k\left(x, x_{1}\right) \int \frac{\lambda \chi(\lambda)\left|\omega_{-}\left(\lambda\left|y-y_{1}\right|\right)\right| \widetilde{\chi}\left(\lambda\left|y-y_{1}\right|\right)}{\delta^{2}+\left|\lambda-\lambda_{0}\right|^{2}} d \lambda \\
& \lesssim k\left(x, x_{1}\right)\left|y-y_{1}\right|^{-\frac{1}{2}} \int_{c\left|y-y_{1}\right|^{-1}}^{1} \frac{\sqrt{\lambda}}{\delta^{2}+\left|\lambda-\lambda_{0}\right|^{2}} d \lambda
\end{aligned}
$$

Now suppose first that $\lambda_{0} \gtrsim \delta$, which is the same as $\left|y-y_{1}\right| \delta \gtrsim 1$. Then

$$
\begin{aligned}
& \left|y-y_{1}\right|^{-\frac{1}{2}} \int_{c\left|y-y_{1}\right|^{-1}}^{1} \frac{\sqrt{\lambda}}{\delta^{2}+\left|\lambda-\lambda_{0}\right|^{2}} d \lambda \\
& \lesssim\left|y-y_{1}\right|^{-\frac{1}{2}}\left\{\int_{0}^{1} \frac{\sqrt{\lambda_{0}}}{\delta^{2}+\left|\lambda-\lambda_{0}\right|^{2}} d \lambda+\int_{0}^{1} \frac{\sqrt{\left|\lambda-\lambda_{0}\right|}}{\delta^{2}+\left|\lambda-\lambda_{0}\right|^{2}} d \lambda\right\} \\
& \lesssim\left|y-y_{1}\right|^{-\frac{1}{2}}\left\{\sqrt{\lambda_{0}} \delta^{-1}+\delta^{-\frac{1}{2}}\right\} \lesssim 1+\left(\left|y-y_{1}\right| \delta\right)^{-1} \lesssim 1,
\end{aligned}
$$


as desired. On the other hand, if $\lambda_{0} \ll \delta$, then also $\left|y-y_{1}\right| \delta \ll 1$ and thus

$$
\begin{gathered}
\left|y-y_{1}\right|^{-\frac{1}{2}} \int_{c\left|y-y_{1}\right|^{-1}}^{1} \frac{\sqrt{\lambda}}{\delta^{2}+\left|\lambda-\lambda_{0}\right|^{2}} d \lambda \\
\lesssim\left|y-y_{1}\right|^{-\frac{1}{2}} \int_{c\left|y-y_{1}\right|^{-1}}^{1} \lambda^{-\frac{3}{2}} d \lambda \lesssim 1 .
\end{gathered}
$$

It remains to bound the contribution of the term involving $a^{\prime}(\lambda)$ in (58). Inspection of (52) reveals that $\left|\partial_{\lambda} F\left(\lambda, x, x_{1}\right)\right| \lesssim \lambda^{-1}$. Combining this with (53) yields

$$
\begin{gathered}
\left|a^{\prime}(\lambda)\right| \lesssim k\left(x, x_{1}\right)\left[\left(\chi(\lambda)+\lambda\left|\chi^{\prime}(\lambda)\right|\right)\left(\lambda\left|y-y_{1}\right|\right)^{-\frac{1}{2}} \widetilde{\chi}\left(\lambda\left|y-y_{1}\right|\right)\right. \\
\left.+\chi(\lambda)\left|\chi^{\prime}\left(\lambda\left|y-y_{1}\right|\right)\right|\right] .
\end{gathered}
$$

We start with the second term in (60). Its contribution to the integral in (58) is

$$
\lesssim \int_{0}^{1} \chi_{\left[\left|\lambda-\lambda_{0}\right|>\delta\right]} \frac{\left|\chi^{\prime}\left(\lambda\left|y-y_{1}\right|\right)\right|}{\left|\lambda-\lambda_{0}\right|} d \lambda
$$

The integration region here is contained inside an interval of the form $\left[c_{1} \mid y-\right.$ $\left.\left.y_{1}\right|^{-1}, c_{2}\left|y-y_{1}\right|^{-1}\right]$ where $c_{1}, c_{2}$ are some positive constants. If $\lambda_{0} \asymp\left|y-y_{1}\right|^{-1}$, then also $\left|y-y_{1}\right| \delta \asymp 1$. Hence in this case

$$
\text { (61) } \lesssim \log \left(1+\delta^{-1}\left|y-y_{1}\right|^{-1}\right) \lesssim 1
$$

If on the other hand either $\lambda_{0} \gg\left|y-y_{1}\right|^{-1}$, or $\lambda_{0} \ll\left|y-y_{1}\right|^{-1}$, then

$$
\text { (61) } \lesssim\left|\log \left(\frac{c_{2}\left|y-y_{1}\right|^{-1}-\lambda_{0}}{c_{1}\left|y-y_{1}\right|^{-1}-\lambda_{0}}\right)\right| \lesssim 1
$$

It remains to consider the first term in (60). Its contribution to the integral in (58) is

$$
\lesssim\left|y-y_{1}\right|^{-\frac{1}{2}} \int_{\left[\lambda\left|y-y_{1}\right| \gtrsim 1\right]} \chi_{\left[\left|\lambda-\lambda_{0}\right|>\delta\right]} \frac{d \lambda}{\left|\lambda-\lambda_{0}\right| \sqrt{\lambda}}
$$

If $\lambda_{0} \ll\left|y-y_{1}\right|^{-1}$, then

$$
\text { (62) } \lesssim\left|y-y_{1}\right|^{-\frac{1}{2}} \int_{\left[\lambda\left|y-y_{1}\right| \gtrsim 1\right]} \frac{d \lambda}{\lambda^{\frac{3}{2}}} \lesssim 1
$$

If, on the other hand, $\lambda_{0} \gtrsim\left|y-y_{1}\right|^{-1}$, then

$$
\begin{aligned}
\text { (62) } & \lesssim\left|y-y_{1}\right|^{-\frac{1}{2}}\left\{\int_{0}^{\frac{1}{2} \lambda_{0}} \frac{d \lambda}{\lambda_{0} \sqrt{\lambda}}+\int_{\frac{1}{2} \lambda_{0}}^{1} \chi_{\left[\left|\lambda-\lambda_{0}\right|>\delta\right]} \frac{d \lambda}{\left|\lambda-\lambda_{0}\right|^{\frac{3}{2}}}\right\} \\
& \lesssim\left|y-y_{1}\right|^{-\frac{1}{2}}\left(\lambda_{0}^{-\frac{1}{2}}+\delta^{-\frac{1}{2}}\right) \lesssim 1
\end{aligned}
$$


as desired. In the last line we used that $\lambda_{0} \gtrsim\left|y-y_{1}\right|^{-1}$ is the same as $\left|y-y_{1}\right| \delta \gtrsim 1$. This concludes the proof of claim (56).

It remains to consider the phase $\phi_{+}(\lambda)=\lambda^{2}+t^{-1}\left|y-y_{1}\right| \lambda$. The corresponding estimate is

$$
\begin{aligned}
& \mid \int_{0}^{\infty} e^{i t \phi_{+}(\lambda)} \lambda \chi(\lambda)\left[\chi\left(\lambda\left|x-x_{1}\right|\right) Y_{0}\left(\lambda\left|x-x_{1}\right|\right)-\frac{2}{\pi} \chi(\lambda(1+|x|)) \log (\lambda(1+|x|))\right] \\
& \left.\omega_{+}\left(\lambda\left|y_{1}-y\right|\right) \tilde{\chi}\left(\lambda\left|y_{1}-y\right|\right) d \lambda|\leq C| t\right|^{-1} k\left(x, x_{1}\right)
\end{aligned}
$$

for all $x, x_{1}, y, y_{1} \in \mathbb{R}^{2}$. Setting

$$
a(\lambda):=\lambda \chi(\lambda) \omega_{+}\left(\lambda \mid y-y_{1}\right) \tilde{\chi}\left(\lambda\left|y-y_{1}\right|\right) F\left(\lambda, x, x_{1}\right)
$$

a single integration by parts in the left-hand side of (63) yields

$$
\text { (63) } \lesssim|t|^{-1} \int_{0}^{\infty} \frac{|a(\lambda)|}{\left|\phi_{+}^{\prime}(\lambda)\right|^{2}} d \lambda+|t|^{-1} \int_{0}^{\infty} \frac{\left|a^{\prime}(\lambda)\right|}{\left|\phi_{+}^{\prime}(\lambda)\right|} d \lambda .
$$

As before, $\lambda_{0}=\frac{\left|y-y_{1}\right|}{2 t}$. Then

$$
\begin{aligned}
\int_{0}^{\infty} \frac{|a(\lambda)|}{\left|\phi_{+}^{\prime}(\lambda)\right|^{2}} d \lambda & \lesssim k\left(x, x_{1}\right)\left|y-y_{1}\right|^{-\frac{1}{2}} \int_{0}^{\infty} \lambda^{\frac{1}{2}}\left(\lambda^{2}+\lambda_{0}^{2}\right)^{-1} \chi_{\left[\lambda\left|y-y_{1}\right| \gtrsim 1\right]} d \lambda \\
& \lesssim k\left(x, x_{1}\right) \int_{0}^{\infty} \lambda^{-\frac{3}{2}}\left|y-y_{1}\right|^{-\frac{1}{2}} \chi_{\left[\lambda\left|y-y_{1}\right| \gtrsim 1\right]} d \lambda \\
& \lesssim k\left(x, x_{1}\right) .
\end{aligned}
$$

To estimate the second integral in (65), we use (60) which remains valid with $\omega_{+}$. Hence

$$
\begin{aligned}
\int_{0}^{\infty} \frac{\left|a^{\prime}(\lambda)\right|}{\left|\phi_{+}^{\prime}(\lambda)\right|} d \lambda & \lesssim k\left(x, x_{1}\right)\left|y-y_{1}\right|^{-\frac{1}{2}} \int_{0}^{\infty} \lambda^{-\frac{1}{2}}\left(\lambda+\lambda_{0}\right)^{-1} \chi_{\left[\lambda\left|y-y_{1}\right| \gtrsim 1\right]} d \lambda \\
& \lesssim k\left(x, x_{1}\right) \int_{0}^{\infty} \lambda^{-\frac{3}{2}}\left|y-y_{1}\right|^{-\frac{1}{2}} \chi_{\left[\lambda\left|y-y_{1}\right| \gtrsim 1\right]} d \lambda \\
& \lesssim k\left(x, x_{1}\right) .
\end{aligned}
$$

In view of the preceding, (65) $\lesssim|t|^{-1} k\left(x, x_{1}\right)$. Hence (63) holds and (154) has been proved.

The final statement about interchanging the roles of $\chi$ and $\tilde{\chi}$ is implicit in the previous proof. Indeed, (55) holds equally well for $Y_{0}$ instead of $J_{0}$. Moreover, one replaces $F\left(\lambda, x, x_{1}\right)$ with $G\left(\lambda, y, y_{1}\right)$, see (46), and the bound (53) with the trivial one $0 \leq G \leq 1$. We skip the details.

The final lemma dealing with $Q D_{0} Q$ controls the contributions of those $\lambda$ for which both resolvents on either side of $v Q D_{0} Q v$ are evaluated at arguments of size $\gtrsim 1$. In this case it will be convenient to work with the full kernel of the resolvents, i.e., the Hankel functions without splitting them into $J_{0}$ and $Y_{0}$. 
Lemma 15. Let $\left(Q D_{0} Q\right)(\cdot, \cdot)$ denote the kernel of $Q D_{0} Q$ and set $\tilde{\chi}=1-\chi$. There is the bound

$$
\begin{aligned}
& \mid \int_{\mathbb{R}^{4}} \int_{0}^{\infty} \int_{\mathbb{R}^{4}} e^{i t \lambda^{2}} \lambda \chi(\lambda) \widetilde{\chi}\left(\lambda\left|x-x_{1}\right|\right) H_{0}^{ \pm}\left(\lambda\left|x-x_{1}\right|\right) v\left(x_{1}\right)\left(Q D_{0} Q\right)\left(x_{1}, y_{1}\right) v\left(y_{1}\right) \\
& \left.H_{0}^{ \pm}\left(\lambda\left|y_{1}-y\right|\right) \widetilde{\chi}\left(\lambda\left|y_{1}-y\right|\right) d \lambda f(x) g(y) d x_{1} d y_{1} d x d y|\leq C| t\right|^{-1}\|f\|_{1}\|g\|_{1}
\end{aligned}
$$

with a constant that only depends on $V$.

Proof. One has

$$
H_{0}^{+}(y) \widetilde{\chi}(y)=e^{i y} \omega_{+}(y) \text { and } H_{0}^{-}(y) \widetilde{\chi}(y)=e^{-i y} \omega_{-}(y)
$$

where $\omega_{-}=\overline{\omega_{+}}$, and $\left|\omega_{ \pm}^{(\ell)}(y)\right| \lesssim(1+|y|)^{-\frac{1}{2}-\ell}$ for all $\ell \geq 0$ (the reader should note that we are slightly abusing notation here, since $\omega_{ \pm}$already appeared as the decay factors of $J_{0}$ - but this abuse of notation is of no consequence). Correspondingly, there will be two phases to consider in (66), namely

$$
\phi_{ \pm}(\lambda)=\lambda^{2} \pm \lambda \frac{\left|x-x_{1}\right|+\left|y-y_{1}\right|}{t} .
$$

Set $p=\left|x-x_{1}\right|$ and $q=\left|y-y_{1}\right|$ for simplicity. We may assume that $p>0$ and $q>0$. We claim that

$$
\left|\int_{0}^{\infty} e^{i t \phi_{ \pm}(\lambda)} \lambda \chi(\lambda) \widetilde{\chi}(\lambda p) \omega_{ \pm}(p \lambda) \widetilde{\chi}(\lambda q) \omega_{ \pm}(q \lambda) d \lambda\right| \lesssim|t|^{-1}
$$

uniformly in $p, q>0$. The phase $\phi_{-}$has a critical point at

$$
\lambda_{0}=\frac{p+q}{2 t} .
$$

Let $a_{ \pm}(\lambda)=\lambda \chi(\lambda) \widetilde{\chi}(\lambda p) \omega_{ \pm}(p \lambda) \omega_{ \pm}(q \lambda) \tilde{\chi}(\lambda q)$. Then by Lemma 2

$$
\begin{aligned}
\left|\int_{0}^{\infty} e^{i t \phi_{-}(\lambda)} a_{-}(\lambda) d \lambda\right| \lesssim|t|^{-1} \int_{0}^{\infty} \frac{\left|a_{-}(\lambda)\right|}{\delta^{2}+\left|\lambda-\lambda_{0}\right|^{2}} d \lambda \\
+|t|^{-1} \int_{0}^{\infty} \frac{\left|a_{-}^{\prime}(\lambda)\right|}{\left|\lambda-\lambda_{0}\right|} \chi_{\left[\left|\lambda-\lambda_{0}\right|>\delta\right]} d \lambda
\end{aligned}
$$

The integral involving $a_{-}(\lambda)$ is

$$
\begin{aligned}
& \lesssim(p q)^{-\frac{1}{2}} \int_{c\left(p^{-1}+q^{-1}\right)}^{1} \frac{d \lambda}{\delta^{2}+\left|\lambda-\lambda_{0}\right|^{2}} \\
& \lesssim(p q)^{-\frac{1}{2}}\left(\delta^{-1} \chi_{\left[\lambda_{0} \gtrsim p^{-1}+q^{-1}\right]}+\left(p^{-1}+q^{-1}\right)^{-1}\right) \lesssim 1 .
\end{aligned}
$$

Here we used that $\lambda_{0} \gtrsim p^{-1}+q^{-1}$ is the same as $p q \gtrsim t$ or $p q \delta^{2} \gtrsim 1$, as well as the bound

$$
(p q)^{-\frac{1}{2}}\left(p^{-1}+q^{-1}\right)^{-1}=\frac{\sqrt{p q}}{p+q} \lesssim 1
$$


Since

$$
\left|a_{-}^{\prime}(\lambda)\right| \lesssim(p q)^{-\frac{1}{2}} \lambda^{-1} \chi_{\left[\lambda \gtrsim p^{-1}+q^{-1}\right]} \chi(\lambda),
$$

the integral involving $a_{-}^{\prime}(\lambda)$ in (69) is

$$
\lesssim(p q)^{-\frac{1}{2}} \int_{c\left(p^{-1}+q^{-1}\right)}^{1} \chi_{\left[\left|\lambda-\lambda_{0}\right|>\delta\right]} \frac{d \lambda}{\lambda\left|\lambda-\lambda_{0}\right|} .
$$

Now suppose that $\lambda_{0} \ll \delta$. Then $\left|\lambda-\lambda_{0}\right|>\delta$ implies that $\lambda-\lambda_{0} \gtrsim \lambda$. It follows that

$$
\text { (70) } \lesssim(p q)^{-\frac{1}{2}} \int_{c\left(p^{-1}+q^{-1}\right)}^{1} \frac{d \lambda}{\lambda^{2}} \lesssim(p q)^{-\frac{1}{2}}\left(p^{-1}+q^{-1}\right)^{-1} \lesssim 1 .
$$

On the other hand, if $\lambda_{0} \gtrsim \delta$ which is the same as $(p+q) \delta \gtrsim 1$, then by CauchySchwarz

$$
\begin{aligned}
\text { (701) } & \lesssim(p q)^{-\frac{1}{2}}\left(\int_{c\left(p^{-1}+q^{-1}\right)}^{1} \frac{d \lambda}{\lambda^{2}}\right)^{\frac{1}{2}}\left(\int_{0}^{1} \chi_{\left[\left|\lambda-\lambda_{0}\right|>\delta\right]} \frac{d \lambda}{\left|\lambda-\lambda_{0}\right|^{2}}\right)^{\frac{1}{2}} \\
& \lesssim(p q)^{-\frac{1}{2}}\left(p^{-1}+q^{-1}\right)^{-\frac{1}{2}} \delta^{-\frac{1}{2}}=(\delta(p+q))^{-\frac{1}{2}} \lesssim 1
\end{aligned}
$$

Hence (68) holds for the phase $\phi_{-}$.

We now turn to $\phi_{+}$. By inspection,

$$
\left|a_{+}(\lambda)\right| \lesssim(p q)^{-\frac{1}{2}} \chi_{\left[\lambda \gtrsim p^{-1}+q^{-1}\right]} \text { and }\left|a_{+}^{\prime}(\lambda)\right| \lesssim \lambda^{-1}(p q)^{-\frac{1}{2}} \chi_{\left[\lambda \gtrsim p^{-1}+q^{-1}\right]} .
$$

Integrating by parts therefore leads to

$$
\text { (68) } \begin{aligned}
& \lesssim|t|^{-1} \int_{0}^{\infty} \frac{\left|a_{+}(\lambda)\right|}{\left|\phi_{+}^{\prime}(\lambda)\right|^{2}} d \lambda+|t|^{-1} \int_{0}^{\infty} \frac{\left|a_{+}^{\prime}(\lambda)\right|}{\left|\phi_{+}^{\prime}(\lambda)\right|} d \lambda \\
& \lesssim|t|^{-1}(p q)^{-\frac{1}{2}} \int_{0}^{\infty}\left(\lambda+\lambda_{0}\right)^{-2} \chi_{\left[\lambda \gtrsim p^{-1}+q^{-1}\right]} d \lambda \\
& \quad+|t|^{-1}(p q)^{-\frac{1}{2}} \int_{0}^{\infty} \lambda^{-1}\left(\lambda+\lambda_{0}\right)^{-1} \chi_{\left[\lambda \gtrsim p^{-1}+q^{-1}\right]} d \lambda \\
& \lesssim|t|^{-1}(p q)^{-\frac{1}{2}} \int_{0}^{\infty} \lambda^{-2} \chi_{\left[\lambda \gtrsim p^{-1}+q^{-1}\right]} d \lambda \lesssim|t|^{-1} \frac{\sqrt{p q}}{p+q} \lesssim|t|^{-1},
\end{aligned}
$$

and thus (68) also holds for $\phi_{+}$. We leave the remaining details to the reader.

We now combine Lemmas [13, 14] and 15] to obtain the following lemma. It bounds the contribution of the constant term in the expansion (32), see also (37).

Lemma 16. For all test functions $f, g$ and all $t$ one has

$$
\begin{aligned}
& \left|\int_{0}^{\infty} e^{i t \lambda^{2}} \lambda \chi(\lambda)\left\langle\left[R_{0}^{+}\left(\lambda^{2}\right) v Q D_{0} Q v R_{0}^{+}\left(\lambda^{2}\right)-R_{0}^{-}\left(\lambda^{2}\right) v Q D_{0} Q v R_{0}^{-}\left(\lambda^{2}\right)\right] f, g\right\rangle d \lambda\right| \\
& \lesssim|t|^{-1}\|f\|_{1}\|g\|_{1}
\end{aligned}
$$

with a constant that only depends on $V$. 
Proof. Recall the representation (28) with $H_{0}^{ \pm}(z)=J_{0}(z) \pm i Y_{0}(z)$. Hence,

$$
\begin{aligned}
& R_{0}^{+}\left(\lambda^{2}\right)\left(x, x_{1}\right) R_{0}^{+}\left(\lambda^{2}\right)\left(y_{1}, y\right)-R_{0}^{-}\left(\lambda^{2}\right)\left(x, x_{1}\right) R_{0}^{-}\left(\lambda^{2}\right)\left(y_{1}, y\right) \\
& =-\frac{i}{8}\left(Y_{0}\left(\lambda\left|x-x_{1}\right|\right) J_{0}\left(\lambda\left|y-y_{1}\right|\right)+J_{0}\left(\lambda\left|x-x_{1}\right|\right) Y_{0}\left(\lambda\left|y-y_{1}\right|\right)\right) .
\end{aligned}
$$

In addition, we break up the integration region $(0, \infty)$ by means of the partition $1=\chi(\lambda)+\tilde{\chi}(\lambda)$. More precisely, write each resolvent as

$$
R_{0}^{ \pm}\left(\lambda^{2}\right)\left(x, x_{1}\right)=\chi\left(\lambda\left|x-x_{1}\right|\right) R_{0}^{ \pm}\left(\lambda^{2}\right)\left(x, x_{1}\right)+\tilde{\chi}\left(\lambda\left|x-x_{1}\right|\right) R_{0}^{ \pm}\left(\lambda^{2}\right)\left(x, x_{1}\right) .
$$

This leads to four different terms in (72). Those terms that contain at least one $\chi\left(\lambda\left|x-x_{1}\right|\right)$ or $\chi\left(\lambda\left|y-y_{1}\right|\right)$ we rewrite further using (73). The other term which involves only $\tilde{\chi}$ we leave in terms of Hankel functions. Each of these different combinations is estimated by one of the previous three lemmas.

Next we turn to the term involving $S$ in (37).

Lemma 17. Let $S$ and $h_{ \pm}(\lambda)$ be as in Lemma 9 . Then for all test functions $f, g$ and all $t$ one has

$$
\begin{aligned}
& \left|\int_{0}^{\infty} e^{i t \lambda^{2}} \lambda \chi(\lambda)\left\langle\left[\frac{1}{h_{+}(\lambda)} R_{0}^{+}\left(\lambda^{2}\right) v S v R_{0}^{+}\left(\lambda^{2}\right)-\frac{1}{h_{-}(\lambda)} R_{0}^{-}\left(\lambda^{2}\right) v S v R_{0}^{-}\left(\lambda^{2}\right)\right] f, g\right\rangle d \lambda\right| \\
& \lesssim|t|^{-1}\|f\|_{1}\|g\|_{1}
\end{aligned}
$$

with a constant that only depends on $V$.

Proof. Recall that $S$ is of finite rank, and thus Hilbert-Schmidt. In particular, if $S(x, y)$ denotes the kernel of $S$, then $|S(x, y)|$ is again an $L^{2}$-bounded operator. Hence, one shows as before that (74) reduces to the bound

$$
\begin{gathered}
\mid \int_{0}^{\infty} e^{i t \lambda^{2}} \lambda \chi(\lambda)\left[H_{0}^{+}\left(\lambda\left|x-x_{1}\right|\right) H_{0}^{+}\left(\lambda\left|y_{1}-y\right|\right) h_{+}^{-1}(\lambda)\right. \\
\left.-H_{0}^{-}\left(\lambda\left|x-x_{1}\right|\right) H_{0}^{-}\left(\lambda\left|y_{1}-y\right|\right) h_{-}^{-1}(\lambda)\right] d \lambda \mid \\
\lesssim|t|^{-1}\left(1+\log ^{-}\left|x-x_{1}\right|\right)\left(1+\log ^{-}\left|y-y_{1}\right|\right) .
\end{gathered}
$$

As before, we set $p:=\left|x-x_{1}\right|$ and $q:=\left|y_{1}-y\right|$ for simplicity. We again need to distinguish whether or not the arguments of the Hankel functions are $>1$ or $<1$. This will be accomplished by means of the usual partition of unity $1=\chi+\widetilde{\chi}$. It will also be important to remember that

$$
h_{+}(\lambda)=a \log \lambda+z \text { and } h_{-}(\lambda)=a \log \lambda+\bar{z},
$$

where $a \neq 0$. It is understood that the cut-off $\chi(\lambda)$ in (74) is such that $h_{ \pm}(\lambda) \neq 0$ on the support of $\chi$. One of the four terms in (75) which arises as a combination of 
$\chi$ and $\tilde{\chi}$ is

$$
\begin{aligned}
& \left|\int_{0}^{\infty} e^{i t \lambda^{2}} \lambda \chi(\lambda) \chi(\lambda p) \chi(\lambda q) \frac{J_{0}(\lambda p) J_{0}(\lambda q)-Y_{0}(\lambda p) Y_{0}(\lambda q)}{\left(\log \lambda+c_{1}\right)^{2}+c_{2}^{2}} d \lambda\right| \\
& +\left|\int_{0}^{\infty} e^{i t \lambda^{2}} \lambda \chi(\lambda) \chi(\lambda p) \chi(\lambda q) \frac{\left[J_{0}(\lambda p) Y_{0}(\lambda q)+Y_{0}(\lambda p) J_{0}(\lambda q)\right]\left(\log \lambda+c_{1}\right)}{\left(\log \lambda+c_{1}\right)^{2}+c_{2}^{2}} d \lambda\right| \\
& \quad \lesssim|t|^{-1}\left(1+\log ^{-} p\right)\left(1+\log ^{-} q\right)
\end{aligned}
$$

This is proved by one integration by parts using $\lambda e^{i t \lambda^{2}}=\frac{1}{2 i t} \frac{d}{d \lambda} e^{i t \lambda^{2}}$. In view of (47) the fractions inside of the two integrals take the values $\frac{4}{\pi^{2}}$ and $\frac{4}{\pi}$, respectively, at $\lambda=0$. Thus, the boundary terms contribute $\lesssim|t|^{-1}$ to the integration by parts. It remains to show that

$$
\begin{gathered}
\int_{0}^{\infty}\left|\frac{d}{d \lambda}\left[\chi(\lambda) \chi(\lambda p) \chi(\lambda q) \frac{J_{0}(\lambda p) J_{0}(\lambda q)-Y_{0}(\lambda p) Y_{0}(\lambda q)}{\left(\log \lambda+c_{1}\right)^{2}+c_{2}^{2}}\right]\right| d \lambda \\
+\int_{0}^{\infty}\left|\frac{d}{d \lambda}\left[\chi(\lambda) \chi(\lambda p) \chi(\lambda q) \frac{\left[J_{0}(\lambda p) Y_{0}(\lambda q)+Y_{0}(\lambda p) J_{0}(\lambda q)\right]\left(\log \lambda+c_{1}\right)}{\left(\log \lambda+c_{1}\right)^{2}+c_{2}^{2}}\right]\right| d \lambda \\
\lesssim\left(1+\log ^{-} p\right)\left(1+\log ^{-} q\right)
\end{gathered}
$$

If the derivative falls on $\chi(\lambda)$, then the resulting term is clearly bounded by $\lesssim$ $\left(1+\log ^{-} p\right)\left(1+\log ^{-} q\right)$. On the other hand, suppose it falls on $\chi(\lambda p)$. Then that term contributes

$$
\lesssim \frac{1+\log ^{-}(q / p)}{1+\log ^{+} p} \chi_{[p \gtrsim 1]} \lesssim 1+\log ^{-} q,
$$

and similarly if the derivative falls on $\chi(\lambda q)$. It therefore remains to check that, with $\lambda_{1}=c p^{-1} \wedge c q^{-1} \wedge c(c$ being some small constant $)$

$$
\begin{gathered}
\int_{0}^{\lambda_{1}}\left|\frac{d}{d \lambda}\left[\frac{J_{0}(\lambda p) J_{0}(\lambda q)-Y_{0}(\lambda p) Y_{0}(\lambda q)}{\left(\log \lambda+c_{1}\right)^{2}+c_{2}^{2}}\right]\right| d \lambda \\
+\int_{0}^{\lambda_{1}}\left|\frac{d}{d \lambda}\left[\frac{\left[J_{0}(\lambda p) Y_{0}(\lambda q)+Y_{0}(\lambda p) J_{0}(\lambda q)\right]\left(\log \lambda+c_{1}\right)}{\left(\log \lambda+c_{1}\right)^{2}+c_{2}^{2}}\right]\right| d \lambda \\
\lesssim\left(1+\log ^{-} p\right)\left(1+\log ^{-} q\right) .
\end{gathered}
$$

We start with (77). Recall the expansion (48) for $Y_{0}^{\prime}$. Also, let $n(\lambda)>0$ be such that $n(\lambda)^{2}=\left(\log \lambda+c_{1}\right)^{2}+c_{2}^{2}$. Then clearly $n(\lambda) \sim|\log \lambda|$ and $n^{\prime}(\lambda)=\lambda^{-1}+$ $O\left((\lambda \log \lambda)^{-1}\right)$ as $\lambda \rightarrow 0$. Hence

$$
\begin{gathered}
\text { (77) } \lesssim \int_{0}^{\lambda_{1}} \mid \frac{\lambda^{-1}+p g(\lambda p)}{n(\lambda)^{2}}\left(\log ^{-}(\lambda q)+O(1)\right)+\frac{\lambda^{-1}+q g(\lambda q)}{n(\lambda)^{2}}\left(\log ^{-}(\lambda p)+O(1)\right) \\
+2 \frac{\left(\log ^{-}(\lambda p)+O(1)\right)\left(\log ^{-}(\lambda q)+O(1)\right)}{n(\lambda)^{3}} n^{\prime}(\lambda) \mid d \lambda+1
\end{gathered}
$$

Each of the three terms inside the absolute value contains an expression of the form $\frac{1}{\lambda \log \lambda}$. Since these are not integrable, one needs to check that they cancel. Indeed, 
combining them yields

$$
\frac{2 \log \lambda}{\lambda n(\lambda)^{2}}-\frac{2(\log \lambda)^{2}}{n(\lambda)^{3}} n^{\prime}(\lambda)=O\left(\lambda^{-1}(\log \lambda)^{-2}\right)
$$

which is integrable. Otherwise, we claim that (79) $\lesssim\left(1+\log ^{-} p\right)\left(1+\log ^{-} q\right)$. To see this, observe first that for all $0<\lambda<\lambda_{1}$,

$$
\log ^{-}(\lambda p)=\log ^{-} \lambda+\log ^{-}(p)=-\log (\lambda)-\log (p), \quad \log ^{-}(\lambda q)=-\log (\lambda)-\log (p) .
$$

Hence,

$$
\begin{aligned}
& \left|\int_{0}^{\lambda_{1}} \frac{p g(p \lambda)}{n(\lambda)^{2}}\left(\log ^{-}(\lambda q)+O(1)\right) d \lambda\right| \\
& \lesssim \int_{0}^{\lambda_{1}} \frac{p|g(p \lambda)|}{n(\lambda)^{2}}\left(1+\log ^{-}(\lambda)\right) d \lambda(1+|\log q|) \\
& \lesssim \int_{0}^{p^{-1}} p|g(p \lambda)| d \lambda\left(1+\log ^{-} q\right) \\
& \lesssim 1+\log ^{-} q .
\end{aligned}
$$

To pass to (81), note that if $q \geq 1$, then

$$
\sup _{0<\lambda<\lambda_{1}} \frac{1+\log ^{-}(\lambda)}{n(\lambda)^{2}}(1+\log q) \lesssim \sup _{0<\lambda<q^{-1}} \frac{1+\log ^{-}(\lambda)^{2}}{n(\lambda)^{2}} \lesssim 1,
$$

whereas if $0<q<1$, then

$$
\sup _{0<\lambda<\lambda_{1}} \frac{1+\log ^{-}(\lambda)}{n(\lambda)^{2}}\left(1+\log ^{-} q\right) \lesssim 1+\log ^{-} q .
$$

Furthermore,

$$
\begin{aligned}
& \left|\int_{0}^{\lambda_{1}} \frac{\lambda^{-1}}{n(\lambda)^{2}}\left(\log ^{-}(\lambda q)-\log \lambda+O(1)\right) d \lambda\right| \\
& \lesssim \int_{0}^{c q^{-1} \wedge c} \frac{1}{\lambda(\log \lambda)^{2}} d \lambda(1+|\log q|) \\
& \lesssim \frac{1}{\log ^{-}\left(c q^{-1} \wedge c\right)}(1+|\log q|) \lesssim 1+\log ^{-} q .
\end{aligned}
$$

Finally, we estimate

$$
\begin{aligned}
& \left|\int_{0}^{\lambda_{1}} \frac{\left(\log ^{-}(\lambda p)+O(1)\right)\left(\log ^{-}(\lambda q)+O(1)\right)-(\log \lambda)^{2}}{n(\lambda)^{3}} n^{\prime}(\lambda) d \lambda\right| \\
& \lesssim \int_{0}^{\lambda_{1}} \frac{n^{\prime}(\lambda)}{n(\lambda)^{3}} d \lambda+\int_{0}^{\lambda_{1}} \frac{\log ^{-}(\lambda q)}{n(\lambda)^{3}} n^{\prime}(\lambda) d \lambda+\int_{0}^{\lambda_{1}} \frac{\log ^{-}(\lambda p)}{n(\lambda)^{3}} n^{\prime}(\lambda) d \lambda \\
& +\left|\int_{0}^{\lambda_{1}} \frac{\log ^{-}(\lambda p) \log ^{-}(\lambda q)-(\log \lambda)^{2}}{n(\lambda)^{3}} n^{\prime}(\lambda) d \lambda\right| .
\end{aligned}
$$


By our previous discussion,

$$
\text { (84) } \lesssim 1+\log ^{-} q+\log ^{-} p
$$

On the other hand,

$$
\begin{aligned}
\text { [85]) } & \lesssim \int_{0}^{\lambda_{1}} \frac{|\log p|\left|\log ^{-}(\lambda q)\right|+|\log q|}{\lambda(\log \lambda)^{3}} d \lambda \\
& \lesssim \int_{0}^{c p^{-1} \wedge c} \frac{|\log p|}{\lambda(\log \lambda)^{2}} d \lambda+\int_{0}^{c q^{-1} \wedge c p^{-1} \wedge c} \frac{|\log p||\log q|}{\lambda(\log \lambda)^{3}} d \lambda \\
& +\int_{0}^{c q^{-1} \wedge c} \frac{|\log q|}{\lambda(\log \lambda)^{3}} d \lambda \\
& \lesssim\left(1+\log ^{-} p\right)\left(1+\log ^{-} q\right) .
\end{aligned}
$$

Combining (87), (86), (82), (83) (and their analogues with $p$ and $q$ interchanged), as well as (80) yields that

$$
(79) \lesssim\left(1+\log ^{-} p\right)\left(1+\log ^{-} q\right),
$$

as claimed. As far as (78) is concerned, it will suffice to treat the term involving $J_{0}(\lambda p) Y_{0}(\lambda q)$. This amounts to bounding

$$
\begin{aligned}
\int_{0}^{\lambda_{1}} & \mid \frac{\left(\lambda p^{2}+O\left(\lambda^{3} p^{4}\right)\right)\left(\log ^{-}(\lambda q)+O(1)\right)}{n(\lambda)^{2}}\left(\log \lambda+c_{1}\right) \\
& +\frac{\left(1+O\left(\lambda^{2} p^{2}\right)\right)\left(-\lambda^{-1}+q g(q \lambda)\right)}{n(\lambda)^{2}}\left(\log \lambda+c_{1}\right) \\
& +\frac{\left(1+O\left(\lambda^{2} p^{2}\right)\right)\left(\log ^{-}(\lambda q)+O(1)\right)}{\lambda n(\lambda)^{2}} \\
& -2 \frac{\left(1+O\left(\lambda^{2} p^{2}\right)\right)\left(\log ^{-}(\lambda q)+O(1)\right)}{n(\lambda)^{3}} n^{\prime}(\lambda)\left(\log \lambda+c_{1}\right) \mid d \lambda
\end{aligned}
$$

The first line (88) contributes $\lesssim 1+\log ^{-} q$, as do all the $O$-terms in the other three lines. The remaining expression inside the absolute values is

$$
-2 \frac{\log \lambda}{\lambda n(\lambda)^{2}}+2(\log \lambda)^{2} \frac{n^{\prime}(\lambda)}{n(\lambda)^{3}}=O\left(\lambda^{-1}(\log \lambda)^{-2}\right)
$$

as $\lambda \rightarrow 0$. This establishes (76).

Next we turn to the term containing the product $\widetilde{\chi}(\lambda p) \widetilde{\chi}(\lambda q)$. In analogy with Lemma 15] we work with the Hankel functions rather than $J_{0}, Y_{0}$. Thus we need to show that

$$
\left|\int_{0}^{\infty} e^{i[t \lambda \pm \lambda(p+q)]} \frac{\lambda \chi(\lambda)}{h_{ \pm}(\lambda)} \widetilde{\chi}(\lambda p) \widetilde{\chi}(\lambda q) \omega_{ \pm}(\lambda q) \omega_{ \pm}(\lambda p) d \lambda\right| \lesssim|t|^{-1}
$$


uniformly in $p, q>0$. Up to the factors $h_{ \pm}^{-1}$ this is the same as (68). Combine these factors with the $\lambda$-factor that appears in the integrand. This leads to functions that satisfy

$$
\left|\frac{\lambda}{h_{ \pm}(\lambda)}\right| \lesssim \lambda \text { and }\left|\frac{d}{d \lambda} \frac{\lambda}{h_{ \pm}(\lambda)}\right| \lesssim 1
$$

on the support of $\chi$. Hence all the arguments from the proof of Lemma [15] apply to this case as well, and (90) holds.

It remains to consider terms that contain $\chi(\lambda p) \widetilde{\chi}(\lambda q)$ or $\chi(\lambda q) \widetilde{\chi}(\lambda p)$. These terms are analogous to those in Lemma 14. We claim that

$$
\begin{aligned}
& \left|\int_{0}^{\infty} e^{i t \lambda^{2}} \lambda \chi(\lambda) \widetilde{\chi}(\lambda p) \chi(\lambda q) \frac{J_{0}(\lambda p) J_{0}(\lambda q)-Y_{0}(\lambda p) Y_{0}(\lambda q)}{\left(\log \lambda+c_{1}\right)^{2}+c_{2}^{2}} d \lambda\right| \\
& +\left|\int_{0}^{\infty} e^{i t \lambda^{2}} \lambda \chi(\lambda) \widetilde{\chi}(\lambda p) \chi(\lambda q) \frac{\left[J_{0}(\lambda p) Y_{0}(\lambda q)+Y_{0}(\lambda p) J_{0}(\lambda q)\right]\left(\log \lambda+c_{1}\right)}{\left(\log \lambda+c_{1}\right)^{2}+c_{2}^{2}} d \lambda\right| \\
& \quad \lesssim|t|^{-1}\left(1+\log ^{-} q\right) .
\end{aligned}
$$

Write $J_{0}, Y_{0}$ as

$$
J_{0}(y)=e^{i y} \rho_{+}(y)+e^{-i y} \rho_{-}(y) \text { and } Y_{0}(y)=e^{i y} \sigma_{+}(y)+e^{-i y} \sigma_{-}(y)
$$

where $\rho_{ \pm}, \sigma_{ \pm}$decay like $y^{-\frac{1}{2}}$ together with the natural derivative bounds. Thus (91) is the same as

$$
\begin{aligned}
& \left|\int_{0}^{\infty} e^{i t \psi_{ \pm}(\lambda)} \lambda \chi(\lambda) \widetilde{\chi}(\lambda p) \chi(\lambda q) \frac{\rho_{ \pm}(\lambda p) J_{0}(\lambda q)-\sigma_{ \pm}(\lambda p) Y_{0}(\lambda q)}{\left(\log \lambda+c_{1}\right)^{2}+c_{2}^{2}} d \lambda\right| \\
& +\left|\int_{0}^{\infty} e^{i t \psi_{ \pm}(\lambda)} \lambda \chi(\lambda) \widetilde{\chi}(\lambda p) \chi(\lambda q) \frac{\left[\rho_{ \pm}(\lambda p) Y_{0}(\lambda q)+\sigma_{ \pm}(\lambda p) J_{0}(\lambda q)\right]\left(\log \lambda+c_{1}\right)}{\left(\log \lambda+c_{1}\right)^{2}+c_{2}^{2}} d \lambda\right| \\
& \quad \lesssim|t|^{-1}\left(1+\log ^{-} q\right),
\end{aligned}
$$

where $\psi_{ \pm}(\lambda)=\lambda^{2} \pm \frac{p \lambda}{t}$. The bound (92) can is obtained by means of Lemma 2 In fact, the analysis in Lemma 14 carries over to this case with minor modifications. To see this, note that

$$
\begin{aligned}
& \left|\lambda \chi(\lambda) \widetilde{\chi}(\lambda p) \chi(\lambda q) \frac{\rho_{ \pm}(\lambda p) J_{0}(\lambda q)-\sigma_{ \pm}(\lambda p) Y_{0}(\lambda q)}{\left(\log \lambda+c_{1}\right)^{2}+c_{2}^{2}}\right| \\
& \lesssim \lambda \chi(\lambda) \widetilde{\chi}(\lambda p) \chi(\lambda q)(\lambda p)^{-\frac{1}{2}}\left(1+\log ^{-} q\right)
\end{aligned}
$$

and also

$$
\begin{aligned}
& \left|\lambda \chi(\lambda) \widetilde{\chi}(\lambda p) \chi(\lambda q) \frac{\left[\rho_{ \pm}(\lambda p) Y_{0}(\lambda q)+\sigma_{ \pm}(\lambda p) J_{0}(\lambda q)\right]\left(\log \lambda+c_{1}\right)}{\left(\log \lambda+c_{1}\right)^{2}+c_{2}^{2}}\right| \\
& \lesssim \lambda \chi(\lambda) \widetilde{\chi}(\lambda p) \chi(\lambda q)(\lambda p)^{-\frac{1}{2}}\left(1+\log ^{-} q\right) .
\end{aligned}
$$

And similarly for the derivatives. Since these bounds are the same (or even slightly better) than those satisfied by the functions $a_{ \pm}$in (57) and (64), the analysis of Lemma 14 pertaining to these functions carries over to this case as well, cf. (58), (63), and (65). This finishes the proof. 
In view of Corollary [10, the only remaining piece in the proof of Proposition 11 is that term in the expansion (37) which involves $E^{ \pm}$.

Lemma 18. Let $E^{ \pm}(\lambda)$ be as in Lemma 9 . Then for all test functions $f, g$ and all $t$ one has

$$
\begin{aligned}
& \left|\int_{0}^{\infty} e^{i t \lambda^{2}} \lambda \chi(\lambda)\left\langle\left[R_{0}^{+}\left(\lambda^{2}\right) v E^{+}(\lambda) v R_{0}^{+}\left(\lambda^{2}\right)-R_{0}^{-}\left(\lambda^{2}\right) v E^{-}(\lambda) v R_{0}^{-}\left(\lambda^{2}\right)\right] f, g\right\rangle d \lambda\right| \\
& \lesssim|t|^{-1}\|f\|_{1}\|g\|_{1}
\end{aligned}
$$

with a constant that only depends on $V$.

Proof. In analogy with Lemmas 13, 14 and 15] we divide the proof into three separate estimates namely,

$$
\begin{aligned}
& \mid \int_{\mathbb{R}^{8}} \int_{0}^{\infty} e^{i t \lambda^{2}} \lambda \chi(\lambda) \chi\left(\lambda\left|x-x_{1}\right|\right) H_{0}^{ \pm}\left(\lambda\left|x-x_{1}\right|\right) v\left(x_{1}\right) E^{ \pm}(\lambda)\left(x_{1}, y_{1}\right) v\left(y_{1}\right) \\
& \left.H_{0}^{ \pm}\left(\lambda\left|y_{1}-y\right|\right) \chi\left(\lambda\left|y_{1}-y\right|\right) d \lambda f(x) g(y) d x_{1} d y_{1} d x d y|\leq C| t\right|^{-1}\|f\|_{1}\|g\|_{1} \\
& \mid \int_{\mathbb{R}^{8}} \int_{0}^{\infty} e^{i t \lambda^{2}} \lambda \chi(\lambda) \chi\left(\lambda\left|x-x_{1}\right|\right) H_{0}^{ \pm}\left(\lambda\left|x-x_{1}\right|\right) v\left(x_{1}\right) E^{ \pm}(\lambda)\left(x_{1}, y_{1}\right) v\left(y_{1}\right) \\
& \left.H_{0}^{ \pm}\left(\lambda\left|y_{1}-y\right|\right) \widetilde{\chi}\left(\lambda\left|y_{1}-y\right|\right) d \lambda f(x) g(y) d x_{1} d y_{1} d x d y|\leq C| t\right|^{-1}\|f\|_{1}\|g\|_{1} \\
& \mid \int_{\mathbb{R}^{4}} \int_{0}^{\infty} \int_{\mathbb{R}^{4}} e^{i t \lambda^{2}} \lambda \chi(\lambda) \widetilde{\chi}\left(\lambda\left|x-x_{1}\right|\right) H_{0}^{ \pm}\left(\lambda\left|x-x_{1}\right|\right) v\left(x_{1}\right) E^{ \pm}(\lambda)\left(x_{1}, y_{1}\right) v\left(y_{1}\right) \\
& \left.H_{0}^{ \pm}\left(\lambda\left|y_{1}-y\right|\right) \widetilde{\chi}\left(\lambda\left|y_{1}-y\right|\right) d \lambda f(x) g(y) d x_{1} d y_{1} d x d y|\leq C| t\right|^{-1}\|f\|_{1}\|g\|_{1} .
\end{aligned}
$$

Unlike in the case of $Q D_{0} Q$ we do not exploit any cancellation between $H_{0}^{+}$and $H_{0}^{-}$. This is not only impossible but also unnecessary. In contrast to $Q D_{0} Q$, the logarithmic singularities of $H_{0}^{ \pm}$at zero are compensated for by the vanishing of $E^{ \pm}(\lambda)$ at $\lambda=0$, see (33).

Let us start with that term where these singularities are not present, i.e., with (98). Set $p=\left|x-x_{1}\right|, q=\left|y-y_{1}\right|$, and $\lambda_{0}=\frac{p+q}{2 t}$. Using the representation (67) and Lemma 2] we arrive at

$$
\begin{aligned}
& \left|\int_{0}^{\infty} e^{i\left[t \lambda^{2}-\lambda(p+q)\right]} \lambda \chi(\lambda) \widetilde{\chi}(\lambda p) \omega_{-}(\lambda p) E^{-}(\lambda)\left(x_{1}, y_{1}\right) \widetilde{\chi}(\lambda q) \omega_{-}(\lambda q) d \lambda\right| \\
& \lesssim|t|^{-1}\left\{\int_{0}^{\infty} \frac{\left|a_{-}(\lambda)\right|}{\delta^{2}+\left|\lambda-\lambda_{0}\right|^{2}} d \lambda+\int_{0}^{\infty} \chi_{\left[\left|\lambda-\lambda_{0}\right|>\delta\right]} \frac{\left|a_{-}^{\prime}(\lambda)\right|}{\left|\lambda-\lambda_{0}\right|} d \lambda\right\} \sup _{0<\lambda<\lambda_{1}}\left|E^{-}(\lambda)\left(x_{1}, y_{1}\right)\right| \\
& +|t|^{-1} \int_{0}^{\infty} \chi_{\left[\left|\lambda-\lambda_{0}\right|>\delta\right]} \frac{\lambda^{-\frac{1}{2}}\left|a_{-}(\lambda)\right|}{\left|\lambda-\lambda_{0}\right|} d \lambda \sup _{0<\lambda<\lambda_{1}} \sqrt{\lambda}\left|\partial_{\lambda} E^{-}(\lambda)\left(x_{1}, y_{1}\right)\right|
\end{aligned}
$$

where we have set

$$
a_{-}(\lambda):=\lambda \chi(\lambda) \widetilde{\chi}(\lambda p) \omega_{-}(\lambda p) \widetilde{\chi}(\lambda q) \omega_{-}(\lambda q)
$$


Note that the first two integrals involving $a_{-}$appearing in (99) have already been treated in Lemma 15. Thus, the expression in braces is $\lesssim 1$. Moreover, the third integral which involves the new term $\lambda^{-\frac{1}{2}} a_{-}(\lambda)$ is actually better than $a_{-}^{\prime}(\lambda)$, since the latter involves the loss of a full power of $\lambda$ relative to $a_{-}$rather than just a half power. Referring to the proof of Lemma 14 we can therefore again claim that the third integral in (99) is $\lesssim 1$. All that remains now is to observe that (98) follows from the preceding by means of the error estimates (33).

The case of $E^{+}$is treated in an analogous fashion, see (71), and we skip the details. Next we consider the other extreme case, i.e., (96) in which $H_{0}^{ \pm}$is only evaluated on the interval $(0,1]$. Setting

$$
a_{ \pm}(\lambda):=\chi(\lambda) \chi(\lambda p) \omega_{ \pm}(\lambda p) \chi(\lambda q) \omega_{ \pm}(\lambda q)
$$

a single integration by parts now yields

$$
\begin{aligned}
& \left|\int_{0}^{\infty} e^{i t \lambda^{2}} \lambda \chi(\lambda) \chi(\lambda p) \omega_{ \pm}(\lambda p) E^{ \pm}(\lambda)\left(x_{1}, y_{1}\right) \chi(\lambda q) \omega_{ \pm}(\lambda q) d \lambda\right| \\
& \lesssim|t|^{-1} \int_{0}^{\infty} \sqrt{\lambda}\left|a_{ \pm}^{\prime}(\lambda)\right| d \lambda \sup _{0<\lambda<\lambda_{1}} \lambda^{-\frac{1}{2}}\left|E^{ \pm}(\lambda)\left(x_{1}, y_{1}\right)\right| \\
& +|t|^{-1} \int_{0}^{\infty} \lambda^{-\frac{1}{2}}\left|a_{ \pm}(\lambda)\right| d \lambda \sup _{0<\lambda<\lambda_{1}} \sqrt{\lambda}\left|\partial_{\lambda} E^{ \pm}(\lambda)\left(x_{1}, y_{1}\right)\right| .
\end{aligned}
$$

Now

$$
\begin{aligned}
& \left|a_{ \pm}(\lambda)\right| \lesssim \chi(\lambda)\left(1+|\log \lambda|^{2}\right)\left(1+\log ^{-} p\right)\left(1+\log ^{-} q\right) \\
& \left|a_{ \pm}^{\prime}(\lambda)\right| \lesssim \chi_{[0<\lambda<1]} \lambda^{-1}(1+|\log \lambda|)\left(1+\log ^{-} p\right)\left(1+\log ^{-} q\right) .
\end{aligned}
$$

To obtain (96), insert these bounds into (100) and invoke (33).

It remains to consider the term of mixed type, i.e., (97). Thus set

$$
a_{-}(\lambda):=\lambda \chi(\lambda) \widetilde{\chi}(\lambda p) \omega_{-}(\lambda p) \chi(\lambda q) \omega_{-}(\lambda q) .
$$

Applying Lemma 2 with $\lambda_{0}=\frac{p}{2 t}$ one obtains

$$
\begin{aligned}
& \left|\int_{0}^{\infty} e^{i\left[t \lambda^{2}-\lambda p\right]} \lambda \chi(\lambda) \widetilde{\chi}(\lambda p) \omega_{-}(\lambda p) E^{-}(\lambda)\left(x_{1}, y_{1}\right) \chi(\lambda q) \omega_{-}(\lambda q) d \lambda\right| \\
& \lesssim|t|^{-1}\left\{\int_{0}^{\infty} \frac{\sqrt{\lambda}\left|a_{-}(\lambda)\right|}{\delta^{2}+\left|\lambda-\lambda_{0}\right|^{2}} d \lambda\right. \\
& \left.+\int_{0}^{\infty} \chi_{\left[\left|\lambda-\lambda_{0}\right|>\delta\right]} \frac{\sqrt{\lambda}\left|a_{-}^{\prime}(\lambda)\right|}{\left|\lambda-\lambda_{0}\right|} d \lambda\right\} \sup _{0<\lambda<\lambda_{1}} \lambda^{-\frac{1}{2}}\left|E^{-}(\lambda)\left(x_{1}, y_{1}\right)\right| \\
& +|t|^{-1} \int_{0}^{\infty} \chi_{\left[\left|\lambda-\lambda_{0}\right|>\delta\right]} \frac{\lambda^{-\frac{1}{2}}\left|a_{-}(\lambda)\right|}{\left|\lambda-\lambda_{0}\right|} d \lambda \sup _{0<\lambda<\lambda_{1}} \sqrt{\lambda}\left|\partial_{\lambda} E^{-}(\lambda)\left(x_{1}, y_{1}\right)\right| .
\end{aligned}
$$


The basic estimates on $a_{-}(\lambda)$ are

$$
\begin{aligned}
& \left|a_{-}(\lambda)\right| \lesssim \lambda \chi(\lambda)(\lambda p)^{-\frac{1}{2}}\left(1+\log ^{-} \lambda\right)\left(1+\log ^{-} q\right) \\
& \left|a_{-}^{\prime}(\lambda)\right| \lesssim \chi_{[0<\lambda<1]}(\lambda p)^{-\frac{1}{2}}\left(1+\log ^{-} \lambda\right)\left(1+\log ^{-} q\right) .
\end{aligned}
$$

Hence

$$
\begin{aligned}
\sqrt{\lambda}\left|a_{-}(\lambda)\right| & \lesssim \lambda \chi(\lambda)(\lambda p)^{-\frac{1}{2}}\left(1+\log ^{-} q\right) \\
\sqrt{\lambda}\left|a_{-}^{\prime}(\lambda)\right|+\lambda^{-\frac{1}{2}}\left|a_{-}(\lambda)\right| & \lesssim \chi_{[0<\lambda<1]}(\lambda p)^{-\frac{1}{2}}\left(1+\log ^{-} q\right) .
\end{aligned}
$$

These are precisely the bounds that were used in the proof of Lemma 14, and one can therefore repeat the arguments appearing there, see (58) to (62). Finally, the phase $t \lambda^{2}+\lambda p$ can be treated as in (65), and we skip the details.

Acknowledgement: The author wishes to thank Monica Visan for comments on a preliminary version of this paper, as well the anonymous referee for a very careful reading and many helpful comments.

\section{References}

[Agm] Agmon, S. Spectral properties of Schrödinger operators and scattering theory. Ann. Scuola Norm. Sup. Pisa Cl. Sci. (4) 2 (1975), no. 2, 151-218.

[GolSch] Goldberg, M., Schlag, W. Dispersive estimates for Schrödinger operators in dimensions one and three. To appear in Comm. Math. Phys., preprint 2003.

[JenNen] Jensen, A., Nenciu, G. A unified approach to resolvent expansions at thresholds. Rev. Math. Phys. 13 (2001), no. 6, 717-754.

[JenYaj] Jensen, A., Yajima, K. A remark on $L^{p}$-boundedness of wave operators for two-dimensional Schrdinger operators. Comm. Math. Phys. 225 (2002), no. 3, 633-637.

[JouSofSog] Journé, J.-L., Soffer, A., Sogge, C. D. Decay estimates for Schrödinger operators. Comm. Pure Appl. Math. 44 (1991), no. 5, 573-604.

[Mur] Murata, M. Asymptotic expansions in time for solutions of Schrödinger-type equations J. Funct. Anal. 49 (1) (1982), 10-56.

[RodSch] Rodnianski, I., Schlag, W. Time decay for solutions of Schrödinger equations with rough and time-dependent potentials. Invent. Math. 155 (2004), 451513.

[Sto] Stoiciu, M. An estimate for the number of bound states of the Schrödinger operator in two dimensions. preprint 2003, Caltech. 
[Wed] Weder, R. $L^{p}-L^{\dot{p}}$ estimates for the Schrödinger equation on the line and inverse scattering for the nonlinear Schrödinger equation with a potential. J. Funct. Anal. 170 (2000), no. 1, 37-68.

[Yaj] Yajima, K. $L^{p}$-boundedness of wave operators for two-dimensional Schrödinger operators. Comm. Math. Phys. 208 (1999), no. 1, 125-152.

Division of Astronomy, Mathematics, and Physics, 253-37 Caltech, PAsadena, CA 91125, U.S.A.

email: schlag@caltech.edu 